\title{
FINITE-DIMENSIONAL REPRESENTATIONS CONSTRUCTED FROM RANDOM WALKS
}

\author{
ANNA ERSCHLER AND NARUTAKA OZAWA
}

\begin{abstract}
Given a 1-cocycle $b$ with coefficients in an orthogonal representation, we show that every finite dimensional summand of $b$ is cohomologically trivial if and only if $\left\|b\left(X_{n}\right)\right\|^{2} / n$ tends to a constant in probability, where $X_{n}$ is the trajectory of the random walk $(G, \mu)$. As a corollary, we obtain sufficient conditions for $G$ to satisfy Shalom's property $H_{\mathrm{FD}}$. Another application is a convergence to a constant in probability of $\mu^{* n}(e)-\mu^{* n}(g), n \gg m$, normalized by its average with respect to $\mu^{* m}$, for any finitely generated infinite amenable group without infinite virtually abelian quotients. Finally, we show that the harmonic equivariant mapping of $G$ to a Hilbert space obtained as an $U$-ultralimit of normalized $\mu^{* n}-g \mu^{* n}$ can depend on the ultrafilter $U$ for some groups.
\end{abstract}

\section{INTRODUCTION}

Convention. Throughout the paper, $G$ is a compactly generated locally compact group with a distinguished relatively compact symmetric subset $Q$ which contains an open generating neighborhood $e$ of $G$, and $\mu$ is a symmetric probability measure on $G$ that satisfies the following conditions:

- $\mu$ is absolutely continuous with respect to the Haar measure $m$,

- $\inf \left\{\frac{d \mu}{d m}(x): x \in Q\right\}>0$,

- $\int|x|_{G}^{d} d \mu(x)<\infty$ for all $d$.

Here $|x|_{G}:=\min \left\{n: x \in Q^{n}\right\}$ (except that $|e|_{G}:=0$ ). Note that $|\cdot|_{G}$ is a length function, that is, it satisfies $|x|_{G}=\left|x^{-1}\right|_{G}$ and $|x y|_{G} \leq|x|_{G}+|y|_{G}$. Put $B_{G}(r):=\{x \in$ $\left.G:|x|_{G} \leq r\right\}$.

Formulation of the results. Throughout the paper, we will work with real Hilbert spaces and orthogonal representations. This is purely for our convenience and all results (but not the proofs) hold true for complex Hilbert spaces and unitary representations (except that the statement of Theorem 2.4 has to be slightly modified), because any complex Hilbert space $\mathcal{H}_{\mathbb{C}}$ is also a real Hilbert space with the real inner product $(v, w) \mapsto \Re\langle v, w\rangle_{\mathcal{H}_{\mathbb{C}}}$, and any 1-cocycle (defined below) with coefficients in a unitary representation can be regarded as the one with coefficients in the corresponding orthogonal representation.

Let $\pi: G \curvearrowright \mathcal{H}$ be an orthogonal representation on a real Hilbert space $\mathcal{H}$. Recall that a 1-cocycle (or simply a cocycle) is a continuous map $b: G \rightarrow \mathcal{H}$ which satisfies the 1cocycle identity: $b(g x)=b(g)+\pi_{g} b(x)$ for all $g, x \in G$. It is a 1-coboundary if there is $v \in \mathcal{H}$ such that $b(x)=v-\pi_{x} v$ for all $x \in G$. We note that $b$ is a 1-coboundary if and

The work of the fist named author is partially supported by the ERC grant GroIsRan. The work of the second named author is partially supported by JSPS KAKENHI Grant Number 26400114. He also expresses his gratitude to the organizers of the programs "Classification of operator algebras: complexity, rigidity, and dynamics", "Von Neumann Algebras", the Mittag-Leffler Institute, the Hausdorff institute and Ecole Normale, Paris for the hospitality during his visits. The authors would like to thank the referee for helpful remarks. 
only if it is bounded on $G$ (Proposition 2.2.9 in [2]). Every cocycle $b$ satisfies that $b(e)=0$ and $\|b(x)-b(y)\|=\left\|b\left(x^{-1} y\right)\right\| \leq\|b\|_{Q}\left|x^{-1} y\right|_{G}$, where $\|b\|_{Q}:=\sup _{g \in Q}\|b(g)\|<\infty$.

A cocycle $b$ is said to be $\mu$-harmonic (or simply harmonic) if $\int b(g x) d \mu(x)=b(g)$ for all $g$, or equivalently $\int b(x) d \mu(x)=0$. Any cocycle $b$ gives rise to an affine isometric action $A: G \times \mathcal{H} \rightarrow \mathcal{H}$ by $A(g, v)=\pi_{g} v+b(g)$ (see Chapter 2 in [2]). Conversely, for any (affine) isometric action on a Hilbert space and a point $v \in \mathcal{H}$, the map $b(g)=A(g, v)-v$ defines a 1-cocycle, and harmonicity of this cocycle is same as harmonicity of the orbit map $g \mapsto A(g, v)$. Under an appropriate assumption on the decay of a non-degenerate measure $\mu$, it is known that a compactly generated locally compact group $G$ admits a non-zero $\mu$ harmonic cocycle with respect to some orthogonal representation if and only if $G$ does not satisfy Kazhdan' property (T). Existence of a non-zero harmonic cocycle on groups which do not satisfy property (T) is proved by Mok ([25, Cor. 0.1]), Korevaar and Schoen [22, Thm 4.1.2] for finitely presented groups (and not discrete definition of harmonicity) and in general case (and discrete definition of harmonicity) by Shalom in [32, Thm 6.1]. We will give somewhat more constructive proof of this fact in Section 4 See also Gromov [14, Section 3.6], [15, Section 7A] Fisher and Margulis [11], Lee and Peres [23, Thm 3.8], Ozawa [29] as well as the book by Bekka, de la Harpe, and Valette [2] for a non-exhaustive list of references about this result.

We say that a 1-cocycle $b$ is finite-dimensional if the $\pi(G)$-invariant subspace $\overline{\operatorname{span}} b(G)$ is finite-dimensional. If $\mathcal{H}=\bigoplus_{i} \mathcal{H}_{i}$ is some orthogonal decomposition of $\mathcal{H}$ into $\pi(G)$ invariant subspaces, then $b=\bigoplus_{i} P_{\mathcal{H}_{i}} b$ is a decomposition of $b$ into 1-cocycles $P_{\mathcal{H}_{i}} b$ (with respect to $\left.\pi\right|_{\mathcal{H}_{i}}$ ). We call each $P_{\mathcal{H}_{i}} b$ a summand of $b$. We say that such summand is cohomologically trivial if it is a 1-coboundary.

Given a probability measure $\mu$ on $G$, let $X_{n}$ denote the trajectory of the random walk $(G, \mu)$, that is, $X_{n}=s_{1} s_{2} \cdots s_{n}$ where increments $s_{i} \in G$ are independent and chosen with respect to $\mu$. The corresponding probability measure and its expectation are denoted by $\mathbb{P}$ and $\mathbb{E}$.

The value of a Hilbert valued $\mu$-harmonic 1-cocycle along a trajectory of the random walk $(G, \mu)$ is a martingale, and therefore

$$
\mathbb{E}\left[\left\|b\left(X_{n}\right)\right\|^{2}\right]=\sum_{k=1}^{n} \mathbb{E}\left[\left\|b\left(X_{k}\right)\right\|^{2}-\left\|b\left(X_{k-1}\right)\right\|^{2}\right]=n \mathbb{E}\left[\left\|b\left(X_{1}\right)\right\|^{2}\right] .
$$

That is, the expected value $\frac{1}{n} \mathbb{E}\left[\left\|b\left(X_{n}\right)\right\|^{2}\right]$ is equal to a constant, not depending on $n$. For any (not necessarily harmonic) 1 -cocycle $b$, the expected value $\frac{1}{n} \mathbb{E}\left[\left\|b\left(X_{n}\right)\right\|^{2}\right]$ has a limit (see Lemma 2.2). Theorem $A$ below characterizes the case when the random variable $\frac{1}{n}\left\|b\left(X_{n}\right)\right\|^{2}$ tends to a constant.

Theorem A. Let $G$ be a compactly generated locally compact group with a probability measure $\mu$ on $G$ as in Convention. Let $b: G \rightarrow \mathcal{H}$ be a 1-cocycle. Then the following conditions are equivalent:

(1) Any finite-dimensional summand of b is cohomologically trivial.

(2) $\frac{1}{n}\left\|b\left(X_{n}\right)\right\|^{2}$ tends to a constant in probability.

Now assume moreover that $b$ is harmonic and put $c=\int_{G}\|b(x)\|^{2} d \mu(x)$. Then the limit

$$
\beta:=\lim _{n \rightarrow \infty} \frac{1}{2 c^{2}} \mathbb{E}\left[\left|\frac{\left\|b\left(X_{n}\right)\right\|^{2}}{n}-c\right|^{2}\right]
$$

always exists, and $\beta=0$ if and only if (1) and (2) hold. If $\beta \neq 0$, then $b$ has a cohomologically non-trivial finite-dimensional summand of dimension $\leq 1 / \beta$. 
A more precise version of Theorem $\triangle$ will be given in Theorem 2.4 where we describe the limit distribution of $\left\|b\left(X_{n}\right)\right\| / \sqrt{n}$. This theorem has the following corollary:

Corollary. Let $b$ be a harmonic cocycle. Then, $b$ is a direct sum of (possibly infinitely many) finite-dimensional cocycles if and only if $\lim \sup _{n} \mathbb{P}\left(\left\|b\left(X_{n}\right)\right\|<c \sqrt{n}\right)>0$ for every $c>0$.

Recall that a group $G$ is said to have Shalom's property $H_{\mathrm{FD}}$ if every orthogonal representation $\pi$ with non-zero reduced cohomology group $\overline{H^{1}}(G, \pi)$ contains a non-zero finite-dimensional subrepresentation. In Corollary 2.5 we show that $G$ satisfies Shalom's property $H_{\mathrm{FD}}$ if at least one of the two following conditions hold: either $\liminf _{n} \| \mu^{* n}-$ $\mu^{*(1+\delta) n} \|_{1}<2$ for some $\delta>0$ or $\limsup _{n} \mu^{* n}\left(B_{G}(c \sqrt{n})\right)>0$ for all $c>0$.

Theorem $\mathrm{A}$ and its corollaries develop the argument from [29]. While the main result of [29] is a new proof of Gromov's polynomial growth theorem, the paper also provides a more general criterion for the property $H_{F D}$ for a finitely generated group in terms of convolutions of random walks is given in Section 4 of [29]. It is shown in [10] that wreath products of $\mathbb{Z}$ with finite groups satisfy the assumption of that criterion, providing examples of groups of super-polynomial growth where the criterion applies. The assumption of the criterion from Section 4 in [29] uses shifted convolution, and it is not clear whether this assumption is defined by an unmarked Cayley graph of $G$. Assume that $(G, \mu)$ is a simple random walk on $G$, that is, $\mu$ is equidistributed on a finite generating set of $G$. The conditions of (1) as well as of (2) of Corollary 2.5 are clearly defined by the unmarked Cayley graph of $G$. We do not know any group which satisfies the assumption of (1) or of (2) of Corollary 2.5 and for which we know that it violates the assumption of Section 4 of [29]. But the conditions of Corollary 2.5 are easier to check than the assumption from [29]. For example, it is easily applicable to solvable Baumslag-Solitar groups, lamplighter groups $\mathbb{Z} \ltimes \bigoplus_{\mathbb{Z}} F$ with $F$ finite, or to polycyclic groups obtained as extension of $\mathbb{Z}^{2}$ by $M \in S L(2, d)$ with eigenvalues of absolute value $\neq 1$. See Section 3 for more examples. We do not know any group which satisfies Shalom's property and does not satisfy the assumption of Corollary 2.5 .

Given a not necessarily harmonic cocycle $b$ on a group without property (T), a harmonic cocycle can be obtained taking averages of $b$ (see Mok, Korevaar Schoen, Shalom [22,25, 31], and in particular this can be achieved averaging with respect to a probability measure $\mu$ (see e.g. Gromov, Lee-Peres [14,23]). In Section 4 we study the cocycles $b_{\mu, U}$, constructed as a ultralimit in $\ell_{2}(G)$ of normalized $\mu^{* n}-g \mu^{* n}$ on a finitely generated amenable group $G$. Kesten's criterion [21] (see also [1]) implies that $\mu^{* n}$ is a sequence of almost invariant vectors in $\ell_{2}(G)$, and one can moreover show (see Theorem 4.3) that the limit is a harmonic 1-cocycle. Applying Theorem A to this 1-cocycle, one obtains

Theorem B. Let $G$ be a finitely generated infinite amenable group without virtually abelian infinite quotients. Let $\mu$ be a finitely-supported symmetric non-degenerate probability measure. Then $\left(\mu^{* 2 n}(e)-\mu^{* 2 n}\left(X_{2 m}\right)\right) / \alpha(m, n)$ tends to a constant in probability $\mu^{* 2 m}$ as $m \rightarrow \infty$ and $n \gg m$. Here $\alpha(m, n)=\mu^{* 2 n}(e)-\mu^{* 2 n+2 m}(e)$ is the average of $\mu^{* 2 n}(e)-\mu^{* 2 n}(g)$ with respect to $\mu^{* 2 m}$. Namely

$$
\lim _{m \rightarrow \infty} \limsup _{n \rightarrow \infty} \mathbb{E}\left|\frac{\mu^{* 2 n}(e)-\mu^{* 2 n}\left(X_{2 m}\right)}{\mu^{* 2 n}(e)-\mu^{* 2 n+2 m}(e)}-1\right|=0 .
$$

Take $n$ much larger than $m$. Observe that a group is amenable if and only if $\mu^{* 2 n}(g) / \mu^{* 2 n}(e)$ is close to 1 in probability with respect to $\mu^{* 2 m}$. Theorem $\mathrm{B}$ gives a sufficient condition for the concentration of the second order term of $\mu^{* 2 n}$. 
Theorem $\mathrm{B}$ applies in particular to any finitely generated amenable torsion group (such as Grigorchuk groups $G_{w}[12]$ ) or to any finitely generated amenable simple group (such as commutator full topological groups of minimal shifts on $\mathbb{Z}$ (which are simple by a result of Matui [24] and amenable by a result of Juschenko-Monod [19]), or to simple groups of intermediate growth constructed recently by Nekrashevych [26]. If $\mu$ is equidistributed on a finite generating set of $G$, then the assumption of Theorem B depends only on the unmarked Cayley graph of $(G, \mu)$. In particular, the theorem gives a necessary condition for an amenable group to be simple in terms of unmarked Cayley graphs. In general, it is known that the property of being simple can not be defined by the unmarked Cayley graphs, as it is shown by Burger and Mozes [4] (their examples are isometric to product of two trees and they are non-amenable). It is to our knowledge an open problem whether a property of being a torsion group can be verified geometrically.

Geometric group theory tries to recover properties of a group from the word metrics of this group. Given a group $G$, generated by a finite set $S$, its action on a metric space $X$ and a point $x_{0} \in X$, the group $G$ is equipped with two metrics: the word metric $d_{G, S}(g, h)$ as well as $d_{X, x_{0}}(g, h)=d_{X}\left(g x_{0}, h x_{0}\right)$. It seems interesting to study which properties of the action, or of the group $G$, can be recovered from these two metrics. Theorem A as well as Corollary 2.5 provide examples of such situation, for $X$ being a Hilbert space and a group $G$ acting by affine transformations of $X$.

Fix a non-principal ultrafilter $U$ on the natural numbers $\mathbb{N}$. Let $b_{\mu, U}^{p, q}$ be the mapping to a vector space equipped with a metric, constructed as $U$ ultralimit of normalized $\left(\mu^{* n}\right)^{q}-$ $g\left(\mu^{* n}\right)^{q}$, considered as elements of $\ell_{p}(G)$ (see Section 5). This means that we divide $g\left(\mu^{* n}\right)^{q}-\left(\mu^{* n}\right)^{q}$ by the $l_{p}$ norm of this expression, considered as a function on $g$, and then we take the ultralimit with respect to $U$. By the construction, the $l_{p}$ norm of $b_{U, \mu, G}^{p, q}$ is one. We recall that any ultralimit of Hilbert spaces is a Hilbert space, so that for $p=2$ and any $q \geq 0$ we obtain a cocycle with respect to some orthogonal representation of $\mathcal{H}$. In particular, for $q=1$ and $p=2, b_{\mu, U}^{p, q}$ coincides up to a multiplicative constant with the harmonic cocycle $b_{\mu, U}$, studied in the proof of Theorem B in Section 4 In general, for $p \neq 2$, we obtain a cocycle with respect to some isometric representation on an abstract $L_{p}$-space.

In Theorem $\mathrm{C}$ below we show that the cocycles $b_{\mu, U}^{p, q}, p \geq 1, q \geq 0$ (in particular, the harmonic cocycle $b_{\mu, U}$ ) can depend on the choice of a non-principal ultrafilter $U$.

Theorem C. Take $p=1$ or 2 and $q=0,1$, or 2 . For any $D \geq 2$ there exist torsion groups $G_{1}, G_{2}, \ldots, G_{D}$ such that the following holds. Consider finitely supported symmetric nondegenerate measures $\mu_{i}$ on $G_{i}$ and put $G=\prod_{j=1}^{D} G_{i}$ and $\mu=\prod_{j=1}^{D} \mu_{i}$. For each $j=1, \ldots, D$ there exists a non-principal ultrafilter $U$ such that the limiting cocycle $b_{\mu, U}^{p, q}$ factors through $G \rightarrow G_{i}$.

Theorem [C shows in particular that there exist at least $D$ mutually distinct limiting cocycles among $\left\{b_{\mu, U}^{p, q}: U\right\}$, and at least $D$ mutually distinct subgroups among possible kernels of such cocycles. Such groups $G$ admit $g_{1}, g_{2} \in G$ such that the ratio $\left(\mu^{* 2 n}(e)-\right.$ $\left.\mu^{* 2 n}\left(g_{1}\right)\right) /\left(\mu^{* 2 n}(e)-\mu^{* 2 n}\left(g_{2}\right)\right)$ does not have a limit as $n \rightarrow \infty$.

The groups $G_{i}$ are constructed as piecewise automatic groups [9], they can be chosen to be of sub-exponential word growth, but in such a way that for each $j$ the group $G_{j}$ is in some sense very close to a non-amenable group on some scale while on this particular scale it does not happen to other $G_{k}, j \neq k$. The contribution to $b_{\mu, U}^{p, q}$ is mainly from $G_{j}$ on this scale, and the kernel of $b_{\mu, U}^{p, q}$ contains $\prod_{k \neq j} G_{k}$. 
The kernels of cocycles $b_{\mu, U}^{p, q}$ are particular cases of what we call $\ell_{p}$-thin subgroups: this is a natural family of subgroups, related to the shifts $\left(\mu^{* n}\right)^{q}$ (see Definition 5.1), which for $p=2, q=1$ is related to amenability, for $p=q=1$ to Poisson-Furstenberg boundary and for $q=0, p \geq 1$ to growth of groups (see Lemma 5.6), these groups in some situation may depend on $p$ (see Example 5.9) and on the measure $\mu$ (see Remark 5.10).

Since the group $G$ in the statement of the theorem is a torsion group, it does not admit a virtual quotient to an infinite cyclic group. In particular, taking $p=2$ we can apply the conclusion of Theorem $\mathrm{B}$ to $(G, \mu)$ to claim that $\mu^{* n}(e)-\mu^{* n}(g)$, normalised by its average $\alpha(m, n)$ is close to a constant in probability $\mu^{* m}$, for $n \gg m$. In other words, for each $n \gg m \mu^{* m}$ is concentrated on a set where normalized $\mu^{* n}(e)-\mu^{* n}(g)$ is close to its mean value, but in view of C these sets may depend essentially on $n$.

We are grateful to Pierre de la Harpe for comments on the preliminary version of this paper.

\section{HARMONIC COCYCLES AND FINITE-DIMENSIONAL SUMMANDS}

We now recall from Sections 4 and 5 in [16] that the space $Z^{1}(G, \pi)$ of 1-cocycles is a Hilbert space under the norm

$$
\|b\|_{L^{2}(\mu)}:=\left(\int_{G}\|b(x)\|^{2} d \mu(x)\right)^{1 / 2}
$$

and it decomposes into an orthogonal direct sum of approximate 1-coboundaries and $\mu$ harmonic 1-cocycles. We will say $b$ is normalized when $\|b\|_{L^{2}(\mu)}=1$.

Lemma 2.1. The space $Z^{1}(G, \pi)$ of 1-cocycles is a Hilbert space with respect to the norm $\|\cdot\|_{L^{2}(\mu)}$. Moreover the norms $\|\cdot\|_{L^{2}(\mu)}$ and $\|\cdot\|_{Q}$ are equivalent.

Proof. We observe that $Z^{1}(G, \pi)$ is a Banach space w.r.t. the norm $\|\cdot\|_{Q}$ (see [2, Chapter 3]), and that $\|b\|_{L^{2}(\mu)} \leq\left(\int|x|_{G}^{2} d \mu(x)\right)^{1 / 2}\|b\|_{Q}$. The other side inequality follows, via Open Mapping Theorem, from the fact that any measurable locally integrable 1-cocycle into a separable Hilbert space is automatically continuous modulo a null set. However, following [16], we give a more direct proof here. Take an open generating neighborhood $U$ of $e$ such that $U \subset Q$ and an open neighborhood $V$ of $e$ such that $V^{2} \subset U$. We observe that $\left(\int\|b(x)\|^{2} d \mu^{* 2}(x)\right)^{1 / 2} \leq 2\|b\|_{L^{2}(\mu)}$ and $\varepsilon:=\inf _{x \in U V} \frac{d \mu^{* 2}}{d m}(x)>0$. Thus, for every $g \in U$ one has

$$
\begin{aligned}
\|b(g)\|^{2} & =m(V)^{-1} \int_{V}\left\|b(g x)-\pi_{g} b(x)\right\|^{2} d m(x) \\
& \leq 2 m(V)^{-1}\left[\int_{g V}\|b(x)\|^{2} d m(x)+\int_{V}\|b(x)\|^{2} d m(x)\right] \\
& \leq 4 \varepsilon^{-1} m(V)^{-1} \int_{U V}\|b(x)\|^{2} d \mu^{* 2}(x) \\
& \leq 16 \varepsilon^{-1} m(V)^{-1}\|b\|_{L^{2}(\mu)}^{2} .
\end{aligned}
$$

Since there is $N \in \mathbb{N}$ such that $Q \subset U^{N}$, this proves that the norms $\|\cdot\|_{L^{2}(\mu)}$ and $\|\cdot\|_{Q}$ are equivalent, and that $Z^{1}(G, \pi)$ is a Hilbert space w.r.t. the norm $\|\cdot\|_{L^{2}(\mu)}$.

The reduced 1-cohomology group $\overline{H^{1}}(G, \pi):=Z^{1}(G, \pi) / \overline{B^{1}(G, \pi)}$ is defined to be the space $Z^{1}(G, \pi)$ of 1-cocycles modulo the closure of the subspace $B^{1}(G, \pi)$ of 1coboundaries. We note that $\overline{B^{1}(G, \pi)}=B^{1}(G, \pi)$ if $\pi$ is finite-dimensional, by Theorem 1 in [16]. See Chapter 3 in [2] for an introduction to first reduced cohomology groups. 
Thus,

$$
Z^{1}(G, \pi)=\overline{B^{1}(G, \pi)} \oplus B^{1}(G, \pi)^{\perp} \text { and } \overline{H^{1}}(G, \pi) \cong B^{1}(G, \pi)^{\perp} .
$$

We observe that $b \in Z^{1}(G, \pi)$ belongs to $B^{1}(G, \pi)^{\perp}$ if and only if it is $\mu$-harmonic in the sense $\int b(x) d \mu(x)=0$ or equivalently $\int b(g x) d \mu(x)=b(g)$ for all $g \in G$. Indeed, this follows from the identities $b\left(x^{-1}\right)+\pi_{x}^{-1} b(x)=b(e)=0$ and

$$
\int\left\langle b(x), v-\pi_{x} v\right\rangle d \mu(x)=2\left\langle\int b(x) d \mu(x), v\right\rangle .
$$

We note that every summand of a $\mu$-harmonic 1 -cocycle is $\mu$-harmonic and that every non-zero $\mu$-harmonic 1-cocycle is not a 1-coboundary.

We recall the general fact about orthogonal representations. Let $(\pi, \mathcal{H})$ be an orthogonal representation of $G$ and put

$$
T_{0}:=\mathbb{E}\left[\pi\left(X_{1}\right)\right]=\int \pi(g) d \mu(g) .
$$

Then, $T_{0}$ is a self-adjoint contraction on the Hilbert space $\mathcal{H}$ such that $T_{0}^{k}=\mathbb{E}\left[\pi\left(X_{k}\right)\right]$ for every $k$. By strict convexity of a Hilbert space, a vector $v \in \mathcal{H}$ satisfies $T_{0} v=v$ if and only if $\pi_{g} v=v$ for $\mu$-a.e. $g$, which is equivalent to that $v$ is $\pi(G)$-invariant. Thus by spectral theory, the operators $\frac{1}{n} \sum_{k=0}^{n-1} T_{0}^{k}=\mathbb{E}\left[\frac{1}{n} \sum_{k=0}^{n-1} \pi\left(X_{k}\right)\right]$ converge in strong operator topology to the orthogonal projection $P_{0}$ onto the subspace of $\pi(G)$-invariant vectors. One moreover has convergence in probability

$$
\left\|\frac{1}{n} \sum_{n=0}^{n-1} \pi\left(X_{k}\right) v-P_{0} v\right\| \stackrel{\mathbb{P}}{\rightarrow} 0
$$

Indeed, to prove it, one may assume $P_{0}=0$ and in this case

$$
\mathbb{E}\left[\left\|\frac{1}{n} \sum_{n=0}^{n-1} \pi\left(X_{k}\right) v\right\|^{2}\right]=\frac{1}{n^{2}} \sum_{k, l=0}^{n-1}\left\langle T_{0}^{|k-l|} v, v\right\rangle \rightarrow 0 .
$$

Lemma 2.2. For every $b \in Z^{1}(G, \pi)=\overline{B^{1}(G, \pi)} \oplus B^{1}(G, \pi)^{\perp}$, one has

$$
\lim _{n} \frac{1}{n} \mathbb{E}\left[\left\|b\left(X_{n}\right)\right\|^{2}\right]=\left\|b_{\text {harm }}\right\|_{L^{2}(\mu)}^{2},
$$

where $b_{\text {harm }}$ is the $B^{1}(G, \pi)^{\perp}$ summand in the above decomposition. In particular, $b$ is nonzero in $\overline{H^{1}}(G, \pi)$ if and only if $\lim \frac{1}{n} \mathbb{E}\left[\left\|b\left(X_{n}\right)\right\|^{2}\right]>0$.

Proof. Let $T_{0}:=\int \pi(g) d \mu(g)$. If $c \in B^{1}(\pi, \mathcal{H})$ is a 1-coboundary, $c(x)=v-\pi_{x} v$, then for every $n$ one has

$$
\frac{1}{n} \mathbb{E}\left[\left\|c\left(X_{n}\right)\right\|^{2}\right]=\frac{2}{n}\left\langle\left(1-T_{0}^{n}\right) v, v\right\rangle \leq 2\left\langle\left(1-T_{0}\right) v, v\right\rangle=\|c\|_{L^{2}(\mu)}^{2} .
$$

Since $c \mapsto \mathbb{E}\left[\left\|c\left(X_{n}\right)\right\|^{2}\right]$ is norm-continuous by Lemma 2.1, the above inequality holds for all $c \in \overline{B^{1}(G, \pi)}$. Hence, for any $c \in \overline{B^{1}(G, \pi)}$, by approximating it by $c_{m} \in B^{1}(G, \pi)$, one has

$$
\limsup _{n} \frac{1}{n} \mathbb{E}\left[\left\|c\left(X_{n}\right)\right\|^{2}\right]=\limsup _{n} \frac{1}{n} \mathbb{E}\left[\left\|\left(c-c_{m}\right)\left(X_{n}\right)\right\|^{2}\right] \leq\left\|c-c_{m}\right\|_{L^{2}(\mu)}^{2} \rightarrow 0 .
$$

Now let $b=c+b_{\text {harm }} \in \overline{B^{1}(G, \pi)}+B^{1}(G, \pi)^{\perp}$ be given. Note that since $b_{\text {harm }}$ is $\mu^{* n}$-harmonic, it is orthogonal to $c$ in $L^{2}\left(\mu^{* n}\right)$. Consequently, one has

$$
\lim _{n} \frac{1}{n} \mathbb{E}\left[\left\|b\left(X_{n}\right)\right\|^{2}\right]=\lim _{n} \frac{1}{n} \mathbb{E}\left[\left\|c\left(X_{n}\right)\right\|^{2}+\left\|b_{\text {harm }}\left(X_{n}\right)\right\|^{2}\right]=\left\|b_{\text {harm }}\right\|_{L^{2}(\mu)}^{2} .
$$


It is not clear whether $\frac{1}{n^{2}} \mathbb{E}\left[\left\|b\left(X_{n}\right)\right\|^{4}\right]$ is bounded for every 1-cocycle $b$. However, it is the case for any $\mu$-harmonic 1 -cocycle $b$ (cf. Footnote 2 in [23]).

Lemma 2.3. For every d, one has

$$
\sup _{n} \sup _{b} \frac{1}{n^{d}} \mathbb{E}\left[\left\|b\left(X_{n}\right)\right\|^{2 d}\right]<\infty
$$

where the supremum runs over all normalized $\mu$-harmonic 1-cocycles $b$.

Proof. We fix a universal orthogonal representation $(\pi, \mathcal{H})$ and consider the operators $U_{n}$ from the space of $\mu$-harmonic cocycles into $L^{2 d}\left(\mu^{* n} ; \mathcal{H}\right)$, given by $U_{n} b=n^{-1 / 2} b$. Since

$$
\left\|U_{n} b\right\|=\left(\frac{1}{n^{d}} \mathbb{E}\left[\left\|b\left(X_{n}\right)\right\|^{2 d}\right]\right)^{1 / 2 d} \leq n^{1 / 2} \mathbb{E}\left[\left|X_{1}\right|_{G}^{2 d}\right]^{1 / 2 d}\|b\|_{Q}
$$

(by the Hölder inequality $\left(\sum_{i=1}^{n} a_{i}\right)^{2 d} \leq n^{2 d-1} \sum_{i=1}^{n} a_{i}^{2 d}$ for $a_{i} \geq 0$ ), the operators $U_{n}$ are bounded by Lemma 2.1 The lemma claims that $U_{n}$ 's are uniformly bounded. For this, by Principle of Uniform Boundedness, it suffices to show $\sup _{n}\left\|U_{n} b\right\|<\infty$ for each $b$. (The use of PUB can be avoided if one does the following proof more meticulously.) We in fact prove that $\limsup _{n} \frac{1}{n^{d}} \mathbb{E}\left[\left\|b\left(X_{n}\right)\right\|^{2 d}\right] \leq(2 d-1)$ !! for each normalized harmonic cocycle $b$, by induction on $d$. Here $(2 d-1) ! !=\prod_{k=1}^{d}(2 k-1)$. The case $d=1$ is clear. By induction hypothesis and the Cauchy-Schwarz inequality when $k$ is odd, we may assume that there is $C>0$ such that $\mathbb{E}\left[\left\|b\left(X_{n}\right)\right\|^{k}\right] \leq C n^{k / 2}$ for all $k \leq 2(d-1)$. It follows that

$$
\begin{aligned}
& \mathbb{E}\left[\left\|b\left(X_{n}\right)\right\|^{2 d}\right]=\iint\|b(x)-b(y)\|^{2 d} d \mu^{* n-1}(x) d \mu(y) \\
& =\iint\left(\|b(x)\|^{2}-2\langle b(x), b(y)\rangle+\|b(y)\|^{2}\right)^{d} d \mu^{* n-1}(x) d \mu(y) \\
& =\iint\|b(x)\|^{2 d}+\left(\begin{array}{c}
d \\
1
\end{array}\right)\|b(x)\|^{2(d-1)}\|b(y)\|^{2} \\
& \quad+4\left(\begin{array}{l}
d \\
2
\end{array}\right)\|b(x)\|^{2(d-2)}|\langle b(x), b(y)\rangle|^{2} d \mu^{* n-1}(x) d \mu(y)+C^{\prime} n^{(2 d-3) / 2} \\
& \leq \mathbb{E}\left[\left\|b\left(X_{n-1}\right)\right\|^{2 d}\right]+(d+2 d(d-1)) \cdot(2 d-3) ! ! \cdot n^{d-1}+C^{\prime} n^{d-3 / 2} \\
& \leq \cdots \leq \sum_{k=1}^{n}\left((2 d-1) ! ! \cdot d n^{d-1}+C^{\prime} k^{d-3 / 2}\right) \\
& =(2 d-1) ! ! \cdot n^{d}+o\left(n^{d}\right),
\end{aligned}
$$

where $C^{\prime}$ is some constant depending on $d$ but not on $n$. This finishes the proof.

We start the proof of Theorem A Recall that the tensor product Hilbert space $\mathcal{H} \otimes \mathcal{H}$ is canonically identified with the space of Hilbert-Schmidt operators $\mathcal{S}_{2}(\mathcal{H})$ on $\mathcal{H}$ via $v^{\prime} \otimes$ $v \leftrightarrow S_{v^{\prime} \otimes v}$, where $S_{v^{\prime} \otimes v}(u)=\langle u, v\rangle v^{\prime}$. Under this identification, the operators $\pi_{g} \otimes \pi_{g}$ on $\mathcal{H} \otimes \mathcal{H}$ act on $\mathcal{S}_{2}(\mathcal{H})$ by conjugation $\operatorname{Ad} \pi_{g}: S \mapsto \pi_{g} S \pi_{g}^{*}$. Every Hilbert-Schmidt operator is compact and every compact self-adjoint operator $S$ has a unique spectral decomposition $S=\sum_{i} \lambda_{i} E_{i}$ where $\lambda_{i} \in \mathbb{R}$ are the non-zero eigenvalues of $S$ and $E_{i}$ are the finite-rank orthogonal projections onto the corresponding eigenspaces. If $v \in \mathcal{H} \otimes \mathcal{H}$ is $(\pi \otimes \pi)(G)$ invariant, then $S_{v}$ is $\operatorname{Ad} \pi(G)$-invariant and so are the spectral projections $E_{i}$ 's, which means that $E_{i} \mathcal{H}$ are finite-dimensional $\pi(G)$-invariant subspaces.

Now let us consider a 1-cocycle $b: G \rightarrow \mathcal{H}$ and put

$$
w:=\int(b \otimes b)(x) d \mu(x) \in \mathcal{H} \otimes \mathcal{H} \text { and } T:=\int \pi_{g} \otimes \pi_{g} d \mu(g) .
$$


Then, $T$ is a self-adjoint contraction on $\mathcal{H} \otimes \mathcal{H}$, which is positivity preserving as an operator on $\mathcal{S}_{2}(\mathcal{H})$. By the previous discussion, $\frac{1}{n} \sum_{k=0}^{n-1} T^{k}$ converges in strong operator topology to the orthogonal projection $P$ from $\mathcal{H} \otimes \mathcal{H}$ onto the subspace of $(\pi \otimes \pi)(G)$ invariant vectors. In particular, $\frac{1}{n} \sum_{k=0}^{n-1} T^{k} w$ converges to $P w$ in norm and $S_{P w}$ is a positive Hilbert-Schmidt operator which is $\operatorname{Ad} \pi(G)$-invariant. For any $\pi(G)$-invariant closed subspace $\mathcal{K} \subset \mathcal{H}$, one has

$$
P_{\mathcal{K}} S_{P w} P_{\mathcal{K}}=S_{\left(P_{\mathcal{K}} \otimes P_{\mathcal{K}}\right) P w}=S_{P\left(P_{\mathcal{K}} \otimes P_{\mathcal{K}}\right) w}=S_{P w_{\mathcal{K}}}
$$

where $w_{\mathcal{K}}=\int\left(b_{\mathcal{K}} \otimes b_{\mathcal{K}}\right)(x) d \mu(x)$ for the cocycle $b_{\mathcal{K}}=P_{\mathcal{K}} b$. If $b$ is finite-dimensional, then the trace $\operatorname{Tr}$ is norm-continuous and

$$
\operatorname{Tr}\left(S_{P w}\right)=\operatorname{Tr}\left(\lim _{n} \frac{1}{n} \sum_{k=0}^{n-1} S_{T^{k} w}\right)=\operatorname{Tr}\left(S_{w}\right)=\|b\|_{L^{2}(\mu)}^{2} .
$$

In general, one has the spectral decomposition

$$
S_{P w}=\sum_{i} \lambda_{i} E_{i}
$$

where $\lambda_{1}, \lambda_{2}, \ldots$ is a finite or infinite sequence of strictly positive numbers and $E_{i} \mathcal{H}$ 's are finite-dimensional $\pi(G)$-invariant subspaces. Thus for $b_{i}:=E_{i} b$ and $b_{\infty}:=b-\left(\sum_{i} b_{i}\right)$, one has the direct sum decomposition $b=b_{\infty}+\sum_{i} b_{i}$. We claim that each 1-cocycle $b_{i}, i \neq \infty$, is nonzero and that $b_{\infty}$ is weakly mixing in the sense that it does not admit a nonzero finite-dimensional summand anymore. First, put $E_{\infty}:=1-\sum_{i} E_{i}$ and observe that for $w_{i}:=\left(E_{i} \otimes E_{i}\right) w=\int\left(b_{i} \otimes b_{i}\right)(x) d \mu(x)$, one has $S_{P w_{i}}=E_{i} S_{P w} E_{i}=\lambda_{i} E_{i}$, including the case $i=\infty$ and $\lambda_{\infty}:=0$. It follows that $b=b_{\infty} \oplus \sum_{i}^{\oplus} b_{i}$ and $S_{P w}=$ $S_{P w_{\infty}} \oplus \sum_{i}^{\oplus} S_{P w_{i}}$ in accordance with $\mathcal{H}=E_{\infty} \mathcal{H} \oplus \bigoplus_{i} E_{i} \mathcal{H}$. That $S_{P w_{\infty}}=0$ means that $b_{\infty}$ is weakly mixing. Thus $\|P w\| \neq 0$ if and only if $b$ has a nonzero finite-dimensional summand. Moreover, one has

$$
\operatorname{Tr}\left(S_{P w}\right)=\sum_{i} \operatorname{Tr}\left(S_{P w_{i}}\right)=\sum_{i} \lambda_{i} \operatorname{Tr}\left(E_{i}\right)=\sum_{i}\left\|b_{i}\right\|_{L^{2}(\mu)}^{2}
$$

and

$$
\|P w\|^{2}=\operatorname{Tr}\left(S_{P w}^{2}\right)=\sum_{i} \lambda_{i}^{2} \operatorname{Tr}\left(E_{i}\right) .
$$

For the proof of Theorem $\mathrm{A}$, in view of Lemma 2.2 and the fact that any nonzero $\mu$ harmonic 1-cocycle is cohomologically non-trivial, we may assume that the 1-cocycle $b$ is $\mu$-harmonic. For such $b$, we have the following more precise form of Theorem $\mathrm{A}$.

For any $\theta \geq 0$ and any finite or infinite (possibly null) sequence $\sigma_{k}$ of positive numbers, we denote by $\chi\left(\theta, \sigma_{k}\right)$ the distribution of $\sqrt{\theta^{2}+\sum_{k} \sigma_{k}^{2} g_{k}^{2}}$, where $g_{k}$ are independent standard centered Gaussian random variables.

Theorem 2.4. Let $G$ be as in Convention. Let b be a normalized $\mu$-harmonic 1-cocycle. Let $w, P w$, and $S_{P w}=\sum_{i} \lambda_{i} E_{i}$ be as defined in $(*)$ before the formulation of the theorem. Then,

$$
\lim _{n \rightarrow \infty} \frac{1}{2} \mathbb{E}\left[\left|\frac{\left\|b\left(X_{n}\right)\right\|^{2}}{n}-1\right|^{2}\right]=\|P w\|^{2} \leq\left(\min _{i} \operatorname{dim} E_{i} \mathcal{H}\right)^{-1} .
$$

Moreover, the random variables $\frac{1}{\sqrt{n}}\left\|b\left(X_{n}\right)\right\|$ converge in distribution and in moments to $\chi\left(\theta, \sigma_{k}\right)$, where $\theta=\left\|E_{\infty} b\right\|_{L^{2}(\mu)}$, and $\sigma_{k}^{2}$ are positive eigenvalues of $S_{P \text { w }}$ counted with multiplicities (i.e., $\sigma_{k}=\lambda_{i}^{1 / 2}$ for $\sum_{l=1}^{i-1} \operatorname{dim} E_{l} \mathcal{H}<k \leq \sum_{l=1}^{i} \operatorname{dim} E_{l} \mathcal{H}$ ), which satisfy $\theta^{2}+\sum_{k} \sigma_{k}^{2}=\|b\|_{L^{2}(\mu)}^{2}=1$. One has $\theta>0$ if and only if $b$ admits a weakly mixing 
summand; and $\sigma_{k}>0$ for some $k$ if and only if $b$ admits a non-zero finite-dimensional summand.

Proof of Theorem $A$ and Theorem 2.4 Let $b$ be a normalized $\mu$-harmonic 1-cocycle. In the discussion above, we already saw $\|P w\| \neq 0$ if and only if $b$ has a nonzero finitedimensional summand. Moreover the above formula implies

$$
\|P w\|^{2}=\sum_{i} \lambda_{i}^{2} \operatorname{Tr}\left(E_{i}\right) \leq\left(\max _{i} \lambda_{i}\right) \operatorname{Tr}\left(S_{P w}\right) \leq\left(\min _{i} \operatorname{Tr}\left(E_{i}\right)\right)^{-1},
$$

since $\operatorname{Tr}\left(S_{P w}\right)=\sum_{i} \lambda_{i} \operatorname{Tr}\left(E_{i}\right) \leq 1$. Note that $\operatorname{Tr}\left(E_{i}\right)=\operatorname{dim} E_{i} \mathcal{H}$.

Next, we prove that $\mathbb{E}\left[\left|\frac{\left\|b\left(X_{n}\right)\right\|^{2}}{n}-1\right|^{2}\right] \rightarrow 2\langle P w, w\rangle=2\|P w\|^{2}$. Recall that

$$
\begin{aligned}
\int(b \otimes b)(x) d \mu^{* n}(x) & =\iint(b \otimes b)(x y) d \mu^{* n-1}(x) d \mu(y) \\
& =\iint(b \otimes b)(x)+\left(\pi_{x} \otimes \pi_{x}\right)(b \otimes b)(y) d \mu^{* n-1}(x) d \mu(y) \\
& =\int(b \otimes b)(x) d \mu^{* n-1}(x)+T^{n-1} w \\
& =\left(1+T+\cdots+T^{n-1}\right) w,
\end{aligned}
$$

and $\int\|b(x)\|^{2} d \mu^{* n}(x)=n$ (see [23] and [29]). Hence

$$
\begin{aligned}
\mathbb{E} & {\left[\left\|b\left(X_{n}\right)\right\|^{4}\right]=\int\|b(x)\|^{4} d \mu^{* n}(x) } \\
& =\iint\left(\|b(x)-b(y)\|^{2}\right)^{2} d \mu^{* n-1}(x) d \mu(y) \\
& =\iint\left[\|b(x)\|^{4}+4|\langle b(x), b(y)\rangle|^{2}+\|b(y)\|^{4}+2\|b(x)\|^{2}\|b(y)\|^{2}\right] d \mu^{* n-1}(x) d \mu(y) \\
& =\mathbb{E}\left[\left\|b\left(X_{n-1}\right)\right\|^{4}\right]+4\left\langle\sum_{k=0}^{n-2} T^{k} w, w\right\rangle+\mathbb{E}\left[\left\|b\left(X_{1}\right)\right\|^{4}\right]+2(n-1) \\
& =4\left\langle\sum_{k=1}^{n-1}(n-k) T^{k-1} w, w\right\rangle+n \mathbb{E}\left[\left\|b\left(X_{1}\right)\right\|^{4}\right]+n(n-1) \\
& \leq 3 n^{2}+O(n) .
\end{aligned}
$$

By Bounded Convergence Theorem, this implies that

$$
\begin{aligned}
\mathbb{E}\left[\left|\frac{\left\|b\left(X_{n}\right)\right\|^{2}}{n}-1\right|^{2}\right] & =\mathbb{E}\left[\frac{1}{n^{2}}\left\|b\left(X_{n}\right)\right\|^{4}-\frac{2}{n}\left\|b\left(X_{n}\right)\right\|^{2}+1\right] \\
& =\frac{4}{n^{2}}\left\langle\sum_{k=1}^{n-1}(n-k) T^{k-1} w, w\right\rangle+\frac{1}{n}\left(\mathbb{E}\left[\left\|b\left(X_{1}\right)\right\|^{4}\right]-1\right) \\
& \rightarrow 2\langle P w, w\rangle .
\end{aligned}
$$

Now since $\sup _{n} \mathbb{E}\left[\left|\frac{1}{n} \| b\left(X_{n}\right)\right|^{2}-\left.1\right|^{3}\right]<\infty$ by Lemma2.3, the sequence $\frac{1}{n}\left\|b\left(X_{n}\right)\right\|^{2}$ tends to a constant (which is necessarily 1 ) in probability if and only if one has $\mathbb{E}\left[\mid \frac{1}{n}\left\|b\left(X_{n}\right)\right\|^{2}-\right.$ $\left.\left.1\right|^{2}\right] \rightarrow 0$. This completes the proof of Theorem $\mathrm{A}$ and the first part of Theorem 2.4

For the second half of Theorem 2.4, we first note that convergence in distribution and convergence in moments are equivalent in our setting. Indeed, by the moments condition $\sup _{n} \frac{1}{n^{d}} \mathbb{E}\left[\left\|b\left(X_{n}\right)\right\|^{2 d}\right]<\infty$ (Lemma 2.3), convergence in distribution implies that in 
moments (see [3, Corollary 25.12]). And conversely, since the normal distribution and the distributions $\chi\left(\theta, \sigma_{k}\right)$ are uniquely determined by their moments (see [3, Theorem 30.1]), convergence in moments to such a distribution implies that in distribution (see [3, Theorem 30.2]).

We use Martingale Central Limit Theorem (Theorem 35.12 in [3]) to prove that for any $v \in \mathcal{H}$ the random variables $S_{n}:=n^{-1 / 2}\left\langle b\left(X_{n}\right), v\right\rangle$ converge to a normal distribution $N(0, q(v))$ where $q(v)=\left\langle S_{P w} v, v\right\rangle$. Consider the martingale array $S_{n, k}:=$ $n^{-1 / 2}\left\langle b\left(X_{k}\right), v\right\rangle, k=1, \ldots, n$, and put

$$
Y_{n, k}:=S_{n, k}-S_{n, k-1}=n^{-1 / 2}\left\langle\pi\left(X_{k-1}\right) b\left(X_{k-1}^{-1} X_{k}\right), v\right\rangle .
$$

Since $X_{k-1}^{-1} X_{k}$ has the same distribution as $X_{1}$, one has

$$
\begin{aligned}
\sum_{k=1}^{n} \mathbb{E}\left[Y_{n, k}^{2} \| X_{1}, \ldots, X_{k-1}\right] & =\frac{1}{n} \sum_{k=1}^{n}\left\langle(\pi \otimes \pi)\left(X_{k-1}\right) w, v \otimes v\right\rangle \\
& \stackrel{\mathbb{P}}{\rightarrow}\langle P w, v \otimes v\rangle=q(v),
\end{aligned}
$$

and, for every $\varepsilon$,

$$
\sum_{k=1}^{n} \mathbb{E}\left[Y_{n, k}^{2} 1_{\left\{\left|Y_{n, k}\right| \geq \varepsilon\right\}}\right] \leq \mathbb{E}\left[\left\|b\left(X_{1}\right)\right\|^{2}\|v\|^{2} 1_{\left\{\left\|b\left(X_{1}\right)\right\| \geq \varepsilon n^{1 / 2}\right\}}\right] \rightarrow 0 .
$$

This shows that the array $S_{n, k}$ satisfies the assumption of the Martingale CLT [3, Thm 35.12], and we can conclude that $S_{n, n} \Rightarrow N(0, q(v))$ in distribution.

Now recall that $S_{P w}=\sum_{i} \lambda_{i} E_{i}$ and $b=b_{\infty}+\sum b_{i}$, and take an orthonormal basis $\left\{v_{i, j}: j=1, \ldots, \operatorname{Tr}\left(E_{i}\right)\right\}$ of $E_{i} \mathcal{H}$. Then, by the previous paragraph, $n^{-1 / 2}\left\langle b\left(X_{n}\right), v_{i, j}\right\rangle$ converges in distribution to a centered Gaussian random variable $g_{i, j}$ with variance $q\left(v_{i, j}\right)=$ $\lambda_{i}$. Moreover, for any $\beta_{i, j} \in \mathbb{R}$, the random variables $\sum_{i, j} \beta_{i, j} n^{-1 / 2}\left\langle b\left(X_{n}\right), v_{i, j}\right\rangle$ converge in moments to $N\left(0, q\left(\sum_{i, j} \beta_{i, j} v_{i, j}\right)\right)$, where

$$
q\left(\sum_{i, j} \beta_{i, j} v_{i, j}\right)=\sum_{i, j} \beta_{i, j}^{2} \lambda_{i}=\sum_{i, j} \beta_{i, j}^{2} q\left(v_{i, j}\right) .
$$

This means that the family $\left\{\left\langle n^{-1 / 2} b\left(X_{n}\right), v_{i, j}\right\rangle\right\}_{i, j}$ are asymptotically independent as $n \rightarrow$ $\infty$. Thus, for any $k \in \mathbb{N}$, one has

$$
\frac{1}{n}\left\|\sum_{i=1}^{k} b_{i}\left(X_{n}\right)\right\|^{2}=\sum_{i=1}^{k} \sum_{j}\left|n^{-1 / 2}\left\langle b\left(X_{n}\right), v_{i, j}\right\rangle\right|^{2} \Rightarrow \sum_{i=1}^{k} \sum_{j} \lambda_{i} g_{i, j}^{2},
$$

where $g_{i, j}$ are independent standard centered Gaussian random variables. Since

$$
\lim _{k} \sup _{n} \mathbb{E}\left[\left(\frac{1}{n}\left\|\sum_{i>k} b_{i}\left(X_{n}\right)\right\|^{2}\right)^{d}\right] \leq \lim _{k} C_{d}\left\|\sum_{i>k} b_{i}\right\|_{L^{2}(\mu)}^{2 d}=0
$$

where $C_{d}$ is a constant independent of $k$ (by Lemma2.3), one has

$$
\lim _{n} \mathbb{E}\left[\left(\frac{1}{n}\left\|\sum_{i} b_{i}\left(X_{n}\right)\right\|^{2}\right)^{d}\right]=\lim _{k} \lim _{n} \mathbb{E}\left[\left(\frac{1}{n}\left\|\sum_{i=1}^{k} b_{i}\left(X_{n}\right)\right\|^{2}\right)^{d}\right]
$$

for every $d$. Also, since $\frac{1}{n}\left\|b_{\infty}\left(X_{n}\right)\right\|^{2} \rightarrow\left\|b_{\infty}\right\|_{L^{2}(\mu)}^{2}$ in moments by the first half of the proof, one has $\frac{1}{n}\left\|b\left(X_{n}\right)\right\|^{2} \rightarrow\left\|b_{\infty}\right\|_{L^{2}(\mu)}^{2}+\sum_{i, j} \lambda_{i} g_{i, j}^{2} \sim \chi\left(\theta, \sigma_{k}\right)^{2}$ in moments. 
Recall that a group $G$ is said to have Shalom's property $H_{\mathrm{FD}}$ ([31]) if every orthogonal representation $\pi$ with $\overline{H^{1}}(G, \pi) \neq 0$ contains a non-zero finite-dimensional subrepresentation. In other words, $G$ has property $H_{\mathrm{FD}}$ if and only if every $\mu$-harmonic 1-cocycle $b$ decomposes into a (possibly infinite) direct sum of finite-dimensional summands. By Theorem 2.4 the latter happens for $b$ if and only if $\lim _{n} \mu^{* n}(\{x \in G:\|b(x)\| \leq c \sqrt{n}\})>0$ for all $c>0$.

Corollary 2.5. Assume either (1) $\liminf _{n}\left\|\mu^{* n}-\mu^{*(1+\delta) n}\right\|_{1}<2$ for some $\delta>0$ or (2) $\lim \sup _{n} \mu^{* n}\left(B_{G}(c \sqrt{n})\right)>0$ for all $c>0$. Then, $G$ has Shalom's property $H_{\mathrm{FD}}$.

Proof. We prove a stronger statement that if $G$ does not have property $H_{\mathrm{FD}}$, then for every $\delta>0$ there are $c>0$ and a sequence $\left(E_{n}\right)_{n}$ of open subsets in $G$ such that $\mu^{* n}\left(E_{n}\right) \rightarrow 1$ and $\mu^{*(1+\delta) n}\left(B_{G}(c \sqrt{n}) E_{n} B_{G}(c \sqrt{n})\right) \rightarrow 0$.

Suppose that there is $\mu$-harmonic 1-cocycle $b: G \rightarrow \mathcal{H}$ without a non-zero finitedimensional summand. We can assume that this cocycle is normalized. Take any $0<$ $\delta<1$. Put $c:=\left(20\|b\|_{Q}\right)^{-1} \delta$ and

$$
E_{n}:=\left\{x \in G:\|b(x)\|^{2}<(1+\delta / 4) n\right\} .
$$

Then, for every $x \in E_{n}$ and $y, z \in B_{G}(c \sqrt{n})$ one has

$$
\|b(y x z)\|^{2} \leq\|b(x)\|^{2}+2\|b(x)\|\left\|b(y)+\pi_{y x} b(z)\right\|+\left\|b(y)+\pi_{y x} b(z)\right\|^{2}<(1+\delta / 2) n
$$

Hence the result follows from Theorem $\mathrm{A}$

Remark 2.6. By Kingman's subadditive ergodic theorem, the linear rate of escape

$$
\lim _{n} \frac{1}{n}\left|X_{n}(\omega)\right|_{G}=\lim _{n} \frac{1}{n} \mathbb{E}\left|X_{n}\right|_{G}=: l_{\mu}
$$

exists and is constant for a.e. $\omega \in(G, \mu)^{\mathbb{N}}$. Hence either of the conditions (1) and (2) in Corollary 2.5 implies that $l_{\mu}=0$ and in particular that $G$ is amenable ([17]).

Remark 2.7. It is known that $\mathbb{Z} \succ \mathbb{Z}$ does not satisfy property $H_{F D}([31,5.4 .1])$. Shalom shows that any infinite amenable group with $H_{\mathrm{FD}}$ admits a virtual quotient to $\mathbb{Z}$ ([31, 4.3.1]). By Corollary [2.5] any non-degenerate random walk on a group without virutal homomorphisms to $\mathbb{Z}$ (or $\mathbb{Z} \imath \mathbb{Z}$ ) does not satisfy either of the conditions (1) or (2). It is apparently on open problem whether the wreath product $\mathbb{Z}^{2}$ ? $(\mathbb{Z} / 2 \mathbb{Z})$ has property $H_{\mathrm{FD}}$ (see [31, 6.6]); the simple random walk on it does not satisfy either of the conditions (for "switch-walk-switch" random walks it follows from Dvoretzky-Erdös theorem ([7, 18]) that the number of distinct sites of a simple random walk on $\mathbb{Z}^{2}$ visited until the time $n$ is asymptotically equivalent to $\mathrm{cn} / \log (n)$, where $c>0$ is a constant.

\section{MORE ON THE PROPERTY $H_{F D}$}

We elaborate on Corollary 2.5. It says $G$ has property $H_{\mathrm{FD}}$ provided that $(G, \mu)$ satisfies the following property. We say a $\mu$-random walk $X_{n}$ is cautious if

$$
\limsup _{n} \mathbb{P}\left(\max _{k=1, \ldots, n}\left|X_{k}\right|_{G}<c \sqrt{n}\right)>0
$$

for every $c>0$. We look at stability of this property under extension. Let $N$ be a closed normal subgroup of $G$ with a length $|\cdot|_{N}$ which may not be proper. We say $N$ is strictly exponentially distorted in $G$ if there exists a constant $C \geq 1$ such that

$$
\frac{1}{C} \log \left(|h|_{N}+1\right)-C \leq|h|_{G} \leq C \log \left(|h|_{N}+1\right)+C
$$


for all $h \in N$. We will denote by $|\cdot|_{G / N}$ the length induced by the compact generating neighborhood $Q N$ of $e$ in $G / N$.

Proposition 3.1. Let $N \triangleleft G$ be a closed normal subgroup which is strictly exponentially distorted, and let $\bar{\mu}$ be the push-out probability measure of $\mu$ to $G / N$. If $(G / N, \bar{\mu})$ is cautious, then so is $(G, \mu)$ and in particular $G$ has Shalom's property $H_{\mathrm{FD}}$.

Proof. It suffices to show that there is a constant $D \geq 1$ with the following property (cf. [35, Lemma 3.4]). Let $s_{i} \in G$ be such that $\left|s_{i}\right|_{G} \leq 1$ and put $g_{k}:=s_{1} \cdots s_{k} \in G$ and $M_{n}:=\max _{k=1, \ldots, n}\left|g_{k} N\right|_{G / N}$. Then, one has $\max _{k=1, \ldots, n}\left|g_{k}\right|_{G} \leq D\left(M_{n}+\log n+1\right)$. To show such $D$ exists, for each $k$, pick $h_{k} \in N$ such that $\left|g_{k}^{-1} h_{k}\right|_{G}=\left|g_{k}^{-1} N\right|_{G / N} \leq$ $M_{k}$. Then, $\left|h_{k-1}^{-1} h_{k}\right|_{G} \leq 2 M_{k}+1 \leq 3 M_{k}$ and so $\left|h_{k-1}^{-1} h_{k}\right|_{N} \leq \exp \left(4 C M_{k}\right)$. Hence $\left|h_{k}\right|_{N} \leq n \exp \left(4 C M_{n}\right)$ for all $k \leq n$ and so

$$
\max _{k=1, \ldots, n}\left|h_{k}\right|_{N} \leq C \log \left(2 n \exp \left(4 C M_{n}\right)\right) \leq(D-1)\left(M_{n}+\log n+1\right)
$$

for some constant $D \geq 1$. Since $\left|g_{k}^{-1} h_{k}\right|_{G} \leq M_{n}$, we are done.

Shalom ([31, Theorem 1.13]) has shown that polycyclic groups have property $H_{\mathrm{FD}}$ by invoking Delorme's theorem ([6]) that connected solvable Lie groups have the corresponding property, and asked if there is another proof of $H_{\mathrm{FD}}$. It is plausible that all connected solvable groups are cautious. We note that in light of Osin's result ([28]) this problem reduces to the case for connected Lie groups with polynomial volume growth.

Corollary 3.2. Let $K$ be a non-archimedean local field and $\mathbb{Z}^{d} \curvearrowright K^{n}$ be a semi-simple linear action such that the semi-direct product $\mathbb{Z}^{d} \ltimes K^{n}$ is compactly generated. Then, $\mathbb{Z}^{d} \ltimes K^{n}$ has Shalom's property $H_{\mathrm{FD}}$.

Proof. Let $\nu_{0}$ be the standard nearest neighborhood random walk on $\mathbb{Z}^{d}$ and $\nu_{1}$ be a uniform probability measure on the compact subgroup $\{x \in K:|x| \leq 1\}$. Since $\left(\mathbb{Z}^{d}, \nu_{0}\right)$ is cautious, for $\mu=\frac{1}{2}\left(\nu_{0}+\nu_{1}^{\otimes n}\right)$, the random walk $\left(\mathbb{Z}^{d} \ltimes K^{n}, \mu\right)$ is cautious.

\section{HARMONIC COCYCLE $b_{\mu, U}$ CONSTRUCTED FROM DIFFERENCES OF SHIFTS OF $\mu^{* n}$}

In this section, we give a rather "explicit" (although we crucially use a non-principal ultrafilter) construction of a non-zero harmonic cocycle on a group that does not satisfy Kazhdan's property (T). In particular, when $G$ is a discrete finitely generated amenable group, a normalized $\mu$-harmonic cocycle $b_{\mu}$ will be obtained as an ultralimit of the sequence $\mu^{* n}-g \mu^{* n} \in \ell_{2}(G)$ after normalization. Throughout this section, we assume (in addition to Convention) that $\mu$ is compactly supported and $\mu=\mu^{\prime * 2}$ for some symmetric probability measure $\mu^{\prime}$ on $G$.

We fix a non-principal ultrafilter $U$ on $\mathbb{N}$ and denote by $\lim _{U}$ the corresponding ultralimit. Then, the ultrapower Hilbert space $\mathcal{H}^{U}$ of a given Hilbert space $\mathcal{H}$ is defined to be

$$
\mathcal{H}^{U}:=\ell_{\infty}(\mathbb{N} ; \mathcal{H}) /\left\{\left(v_{n}\right)_{n=1}^{\infty}: \lim _{U}\left\|v_{n}\right\|=0\right\}
$$

with the inner product $\left\langle\left[v_{n}^{\prime}\right]_{n},\left[v_{n}\right]_{n}\right\rangle:=\lim _{U}\left\langle v_{n}^{\prime}, v_{n}\right\rangle$, where $\left[v_{n}\right]_{n}$ is the equivalence class of $\left(v_{n}\right)_{n} \in \ell_{\infty}(\mathbb{N} ; \mathcal{H})$. An orthogonal representation $\pi$ of $G$ on $\mathcal{H}$ gives rise to the ultrapower representation $\pi^{U}$ on $\mathcal{H}^{U}$ by $\pi_{g}^{U}\left[v_{n}\right]_{n}=\left[\pi_{g} v_{n}\right]_{n}$. (NB: In general, the ultrapower representation is no longer continuous.) We apply this construction to an orthogonal representation $(\pi, \mathcal{H})$ which admits an approximate invariant vectors but no non-zero invariant vectors. By definition, such an orthogonal representation exists if and only if $G$ does not satisfy Kazhdan's property (T) (see [2]). 
Lemma 4.1. Let $(\pi, \mathcal{H})$ be an orthogonal representation which admits an approximate invariant vectors but no non-zero invariant vectors, and consider the positive and contractive operator $T:=\pi(\mu)$ on $\mathcal{H}$. Then, there is a unit vector $v \in \mathcal{H}$ such that the corresponding probability measure $\nu$ on $[0,1]$, defined by the formula

$$
\int_{0}^{1} t^{n} d \nu(t)=\left\langle T^{n} v, v\right\rangle
$$

satisfies $1 \in \operatorname{supp} \nu$ and $\nu(\{1\})=0$.

Proof. Let $E_{T}$ denote the spectral measure corresponding to the self-adjoint operator $T$. Since $(\pi, \mathcal{H})$ admits approximate invariant vectors, the spectrum of $T$ contains 1 , which means that $E_{T}([1-1 / n, 1]) \neq 0$ for any $n$. Hence, there is a unit vector $v \in \mathcal{H}$ such that $E_{T}([1-1 / n, 1]) v \neq 0$ for any $n$. On the other hand, $E_{T}(\{1\})=0$ since $(\pi, \mathcal{H})$ has no non-zero invariant vectors. The probability measure $\nu(\cdot):=\left\langle E_{T}(\cdot) v, v\right\rangle$ corresponding to $v$ satisfies the desired conditions.

Take $(\pi, \mathcal{H}, v)$ as above and put $T=\pi(\mu)$. In case $G$ is a discrete finitely generated infinite amenable group, one can take $(\pi, \mathcal{H}, v)$ to be $\left(\lambda, \ell_{2}(G), \delta_{e}\right)$ by Kesten's theorem ([21]). Consider the coboundary $c_{n}: G \rightarrow \mathcal{H}$ given by $c_{n}(g)=T^{n / 2} v-\pi(g) T^{n / 2} v$ and its normalization $b_{n}:=\left\|c_{n}\right\|_{L^{2}(\mu)}^{-1} c_{n}$. We note that

$$
\left\|c_{n}\right\|_{L^{2}(\mu)}^{2}=2\left\langle\left(T^{n}-T^{n+1}\right) v, v\right\rangle=2 \int_{0}^{1} t^{n}(1-t) d \nu(t) .
$$

We will define the cocycle $b_{\mu}$ to be the ultralimit of $b_{n}$. For continuity of $b_{\mu}$, we need equi-continuity of $b_{n}$ 's. Observe that for every $g \in G$, one has

$$
c_{n}(g)=-\int_{G}\left(\frac{d \mu}{d m}-g \frac{d \mu}{d m}\right)(x) c_{n-2}(x) d m(x) .
$$

Let $K=Q \operatorname{supp} \mu$ (recall that $Q$ is a relatively compact generating subset of $G$ and that supp $\mu$ is assumed compact) and take a constant $C$ which satisfies $\|c\|_{K} \leq C\|c\|_{L^{2}(\mu)}$ for every cocycle $c$ (see Lemma2.1). Then by the above equality, for every $g \in Q$, one has

$$
\left\|b_{n}(g)\right\| \leq \frac{\left\|c_{n-2}\right\|_{K}}{\left\|c_{n}\right\|_{L^{2}(\mu)}} \cdot\left\|\frac{d \mu}{d m}-g \frac{d \mu}{d m}\right\|_{L^{1}} \leq C \frac{\left\|c_{n-2}\right\|_{L^{2}(\mu)}}{\left\|c_{n}\right\|_{L^{2}(\mu)}} \cdot\left\|\frac{d \mu}{d m}-g \frac{d \mu}{d m}\right\|_{L^{1}} .
$$

Since $\frac{d \mu}{d m} \in L^{1}(G)$, the function $g \mapsto\left\|\frac{d \mu}{d m}-g \frac{d \mu}{d m}\right\|_{L^{1}}$ is continuous. Thus, equi-continuity of $b_{n}$ 's follows from the following auxiliary lemma.

Lemma 4.2. Let $\nu$ be a probability measure on $[0,1]$ such that $1 \in \operatorname{supp} \nu$ and $\nu(\{1\})=0$. Then, $\gamma(n):=\int_{0}^{1} t^{n}(1-t) d \nu(t)$ satisfies $\gamma(n) \searrow 0$ and $\gamma(n+1) / \gamma(n) \nearrow 1$.

Proof. The first assertion is obvious. Since

$$
\gamma(n+1)=\int t^{n / 2}(1-t)^{1 / 2} \cdot n^{(n+2) / 2}(1-t)^{1 / 2} d \nu(t) \leq \gamma(n)^{1 / 2} \gamma(n+2)^{1 / 2},
$$

the sequence $\gamma(n+1) / \gamma(n)$ is increasing and has a limit $\delta \leq 1$. Suppose for a contradiction that $\delta<1$. Then, one has $\gamma(n) \leq C \delta^{n}$ and so $\int_{0}^{1} t^{n} d \nu(t)=\sum_{k=n}^{\infty} \gamma(k) \leq C^{\prime} \delta^{n}$ for every $n$, where $C$ and $C^{\prime}$ are some constant independent of $n$. This implies $\operatorname{supp} \nu \subset[0, \delta]$, a contradiction. Hence $\delta=1$.

Since $b_{n}$ 's are equi-continuous and $\left\|b_{n}(g)\right\| \leq|g|_{G}\left\|b_{n}\right\|_{Q}$ is bounded for each $g$, the formula

$$
b_{\mu}(g):=\left[b_{n}(g)\right]_{n} \in \mathcal{H}^{U}
$$


defines a continuous map such that $b_{\mu}(g h)=b_{\mu}(g)+\pi_{g}^{U} b_{\mu}(h)$. Since $b_{\mu}$ is continuous, the ultrapower orthogonal representation $\pi^{U}$ is continuous when restricted to $\overline{\operatorname{span}} b(G)$. Hence $b_{\mu}$ is a 1-cocycle. It is normalized:

$$
\left\|b_{\mu}\right\|_{L^{2}(\mu)}^{2}=\int \lim _{U}\left\|b_{n}(x)\right\|^{2} d \mu(x)=\lim _{U} \int\left\|b_{n}(x)\right\|^{2} d \mu(x)=1,
$$

where, to interchange the ultralimit and integration, we have used the fact that $\mu$ is compactly supported and $b_{n}$ 's are equi-continuous. The constructed 1-cocycle $b_{\mu}$ may depend on the choice of a non-principle ultrafilter $U$ (see Theorem $\mathbf{C}$ ), and we will write $b_{\mu, U}$ instead of $b_{\mu}$ when we want to emphasize the role of the ultrafilter $U$. The following reproves the results of Mok ([25]), Korevaar-Schoen ([22]), and Shalom ([32]) mentioned in Introduction.

Theorem 4.3. Let $G$ be a compactly generated locally compact group which does not have Kazhdan's property $(\mathrm{T})$ and $\mu,(\pi, \mathcal{H}, v)$, and $b_{\mu}$ be as above. Then, $b_{\mu}$ is a normalized $\mu$-harmonic cocycle.

Proof. It only remains to prove that $b_{\mu}$ is harmonic. Put $\gamma(n)=\int t^{n}(1-t) d \nu(t)$. Then, one has

$$
\left\|\int b_{n}(x) d \mu(x)\right\|^{2}=\frac{\gamma(n)-\gamma(n+1)}{2 \gamma(n)} \rightarrow 0
$$

by Lemma 4.2. Hence, for every $v^{\prime}=\left[v_{n}^{\prime}\right]_{n} \in \mathcal{H}^{U}$, one has

$$
\begin{aligned}
\left\langle\int b_{\mu}(x) d \mu(x), v^{\prime}\right\rangle & =\int \lim _{U}\left\langle b_{n}(x), v_{n}^{\prime}\right\rangle d \mu(x) \\
& =\lim _{U} \int\left\langle b_{n}(x), v_{n}^{\prime}\right\rangle d \mu(x) \\
& =\lim _{U}\left\langle\int b_{n}(x) d \mu(x), v_{n}^{\prime}\right\rangle=0 .
\end{aligned}
$$

This means $\int b_{\mu}(x) d \mu(x)=0$ and $b_{\mu}$ is harmonic.

In case $G$ is a discrete amenable group and $(\pi, \mathcal{H}, v)=\left(\lambda, \ell_{2}(G), \delta_{e}\right)$, a computation yields that

and

$$
\left\|c_{n}\right\|_{L^{2}(\mu)}^{2}=2\left(\mu^{* n}(e)-\mu^{* n+1}(e)\right)
$$

$$
\left\|b_{\mu}(g)\right\|^{2}=\lim _{U}\left\|b_{n}(g)\right\|^{2}=\lim _{U} \frac{\mu^{* n}(e)-\mu^{* n}(g)}{\mu^{* n}(e)-\mu^{* n+1}(e)} .
$$

Proof of Theorem $\mathbb{B}$ By Theorem $\mathrm{A}$ we know that $\mathbb{E}\left[\left|\frac{\left\|c\left(X_{m}\right)\right\|^{2}}{m}-1\right|^{2}\right] \rightarrow 0$ for any normalized harmonic cocycle $c$ without non-zero finite-dimensional summands. We will show that in case $G$ does not admit any non-zero harmonic finite-dimensional cocycle (which is the case when $G$ is a finitely generated amenable group without virtually abelian infinite quotients), this convergence is uniform for normalized harmonic cocycles $c$ on $G$. Indeed, we have seen in the proof of Theorem $\mathrm{A}$ that

$$
\mathbb{E}\left[\left|\frac{\left\|c\left(X_{m}\right)\right\|^{2}}{m}-1\right|^{2}\right] \leq \frac{4}{m^{2}}\left\langle\sum_{k=1}^{m-1}(m-k) T^{k-1} w, w\right\rangle+\frac{1}{m}\|c\|_{Q}^{4} \mathbb{E}\left[\left|X_{1}\right|_{G}^{4}\right] \rightarrow 0
$$

for every normalized $\mu$-harmonic 1-cocycle $c$, where $T=\int(\pi \otimes \bar{\pi})_{g} d \mu(g)$ and $w=$ $\int(c \otimes \bar{c})(g) d \mu(g)$. Note that $\|c\|_{Q}$ is uniformly bounded by Lemma 2.1. Therefore, it suffices to prove that $\lim _{k}\left\|T^{k} w\right\|=0$ uniformly for $c$. Suppose that the latter is not the 
case: there are $\varepsilon>0$, a subsequence $k_{m} \rightarrow \infty$, and normalized harmonic cocycles $c_{m}$ with the corresponding $T_{m}$ and $w_{m}$ such that $\left\|T_{m}^{k_{m}} w_{m}\right\| \geq \varepsilon$ for all $m$. Fix a non-principal ultrafilter $U$ and let $c_{U}$ denote the $U$-ultralimit cocycle of the sequence $\left(c_{m}\right)_{m}$, with the corresponding objects denoted by $T_{U}$ and $w_{U}$. Then, $c_{U}$ is a normalized harmonic cocycle. Moreover since $t^{2 k}$ is decreasing in $k$ for any $t \in[-1,1]$, one has for each $k$

$$
\left\langle T_{U}^{2 k} w_{U}, w_{U}\right\rangle=\lim _{U}\left\langle T_{m}^{2 k} w_{m}, w_{m}\right\rangle \geq \lim _{U}\left\langle T_{m}^{2 k_{m}} w_{m}, w_{m}\right\rangle \geq \varepsilon^{2} .
$$

Let $Q$ denote the spectral projection of $T_{U}$ corresponding to eigenvalues $\{-1,+1\}$. Then,

$$
\left\|Q w_{U}\right\|^{2}=\lim _{k}\left\langle k^{-1}\left(1+T^{2}+T^{4}+\cdots+T^{2(k-1)}\right) w_{U}, w_{U}\right\rangle \geq \varepsilon^{2} .
$$

Since $T_{U}^{2} Q w_{U}=Q w_{U}$, the vector $Q w_{U}$ is invariant under $(\pi \otimes \bar{\pi})_{g}$ for all $g \in \operatorname{supp} \mu^{* 2}$. However since $G_{0}:=\left\langle\operatorname{supp} \mu^{* 2}\right\rangle$ has finite-index in $G$, it does not admit a non-zero $\mu^{* 2}$-harmonic 1-cocycle, which implies that $Q w_{U}=0$ (as discussed in the proof of Theorem $\mathrm{A}$. We have arrived at a contradiction.

It follows that if $G$ satisfies the assumption of Theorem $\mathbb{B}$, then $\mathbb{E}\left[\left|\frac{\left\|c\left(X_{m}\right)\right\|^{2}}{m}-1\right|^{2}\right] \rightarrow 0$ uniformly for normalized $\mu$-harmonic 1-cocycles $c$. In particular,

$$
\lim _{m} \limsup _{n} \mathbb{E}\left[\left|\frac{\left\|b_{n}\left(X_{m}\right)\right\|^{2}}{m}-1\right|^{2}\right]=\limsup _{m} \mathbb{E}\left[\left|\frac{\left\|b_{\mu, U}\left(X_{m}\right)\right\|^{2}}{m}-1\right|^{2}\right]=0 .
$$

(Note that $\lim \sup _{n} \lambda_{n}=\sup _{U} \lim _{U} \lambda_{n}$ for any bounded sequence $\lambda_{n}$.) Since

$$
\frac{1}{m}=\lim _{n} \frac{\mu^{* n}(e)-\mu^{* n+1}(e)}{\mu^{* n}(e)-\mu^{* n+m}(e)}
$$

by Lemma 4.2 this completes the proof of Theorem $B$ (after exchanging $\mu$ with $\mu^{* 2}$ ).

\section{5. $\ell_{p}$-THIN SUBGROUPS}

5.1. Definitions. Take a finitely generated group $G$ equipped with a probability measure $\mu$, and ask again what information about its subgroups and quotient groups one can obtain by looking on the behavior the random walk $(G, \mu)$. To ensure the existence of nontrivial quotients, we may search normal subgroups of $G$ defined by convolutions of $G$. A more general question one can ask is what are possible (not necessarily normal subgroups) defined in such terms.

Definition 5.1. $\left[\ell_{p}\right.$-thin subgroups $\left.H_{\mu, p, q}\right]$. Let $G$ be an infinite group generated by a finite set $S$, and $\mu$ be a probability measure on $G$. Fix some $q \geq 0, p \geq 1$ and a sequence $n_{i}$ tending to $\infty$. Assume that $\mu$ is such that $\left(\mu^{* n}\right)^{q}$ is in $l_{p}(G)$ for all $n$ (this holds for example if $\mu$ has finite support). Let $\alpha(n)$ denotes the maximum of $\ell_{p}$ norm of $\left(\mu^{* n}\right)^{q}-$ $g\left(\mu^{* n}\right)^{q}$, where the maximum is taken over $g \in S$. Consider $g \in G$ for which $\|\left(\mu^{* n_{i}}\right)^{q}-$ $g\left(\mu^{* n_{i}}\right)^{q} \|_{p} / \alpha\left(n_{i}\right) \rightarrow 0$ as $i \rightarrow \infty$. If $G$ contains at least two elements, then by the triangular inequality in $\ell_{p}$, such elements form a subgroup of $G$, which we we call the main $\ell_{p}$-thin subgroup and which we denote by $H_{\mu, p, q, n_{i}}$ (and $H_{\mu, p}$ for short, if $n_{i}$ is specified and $q=1$ ).

Now we define $\ell_{p}$-thin subgroups associated an arbitrary function $\alpha(n)$. Consider $g$ such that $\left\|\left(\mu^{* n}\right)^{q}-g\left(\mu^{* n}\right)^{q}\right\|_{p} / \alpha(n)$ tends to 0 as $n$ tends to infinity. By triangular inequality in $\ell_{p}$ such elements form a subgroup of $G$, which we denote $H_{\mu, p, q, \alpha}$. We call this subgroup $\ell_{p}$-thin subgroup associated to $\alpha(n)$.

Remark 5.2. For $q=0$ in the definition above we use the convention $0^{0}=0$; the $\ell_{1}$ norm in this case is therefore the cardinality of the symmetric differences of the supports of $\mu^{* n}$ and $g \mu^{* n}$, that is the cardinality of the set of points $x$ such that either $x$ is in the support 
of $\mu^{* n}$ and $g x$ is not in this support or vice versa. In the definition we have assumed that $p \geq 1$. We can extend the definition for the case $p=0$, defining $\alpha(n)$ as the maximum of the cardinality of the support of $\left(\mu^{* n}\right)^{q}-g\left(\mu^{* n}\right)^{q}$, where the maximum is taken over $g \in S$. In this case we obtain $H_{0,1, \mu}=H_{1,0, \mu}$ for all $\mu$. Observe that that if the support of $\mu$ is a finite symmetric generating set containing the identity, then the support of $\mu^{* n}$ is the ball of radius $n$ in the word metric associated to $S$.

It is clear that the scaling sequence $\alpha(n)$ depends of a finite generating set $S$ up to multiplication by a constant only, and thus the definition of main $\ell_{p}$-thin subgroups does not depend on the choice of $S$.

In many situation the limit behavior of $\left(\mu^{* n}\right)^{q}-g\left(\mu^{* n}\right)^{q}$ does not depend on the subsequence of possible $n$ 's. However, in some situation this quantity, and the corresponding $\ell_{p}$-thin subgroups may depend on the choice of a subsequence, see Theorem $\mathrm{C}$ and Corollary 5.11

Remark 5.3. If $p \geq 1$, it is known that a normalized sequence $v_{n} \in \ell_{1}(G)$ is almost invariant in $\ell_{1}$ with respect to the shift by some element $g \in G$ if and only if $v_{n}^{1 / p}$ (which is clearly a sequence in $\ell_{p}(G)$ ) is almost invariant in $\ell_{p}$ with respect to the shift by $g$ (see e.g. the proof of Theorem 8.3.2 in [30]. This implies that the main $\ell_{p}$-thin subgroups satisfy $H_{p, 1}=H_{1, p}=H_{p / q, q}$ for any $p, q \geq 1$ whenever $\left(\mu^{* n_{i}}\right)^{p}$ does not admit a subsequence of almost invariant vectors in $\ell_{1}$. This happens for example for $p=2$, if $G$ non-amenable and for $p=1$ if the Poisson boundary of $(G, \mu)$ is non-trivial, for all $n_{i}$ ([20]).

It is possible that the statement of Remark 5.3 remains valid without the assumption of non-almost-invariance.

Instead $\left(\mu^{* n}\right)^{q}$ in the Defintion 5.1 one can consider more generally a sequence of functions $f_{i}$ and consider the difference of corresponding shifted functions, as a function of $g$.

We have already remarked that for $p=2, q=1, \mu$ being equidistributed on a finite symmetric set of $G$, the values of $b_{\mu, U}$ are defined by the unmarked Cayley graph of $G$. In particular, for $p=2, q=1$ and $\mu$ being a measure equidistributed on a finite generating set $S$, the $\ell_{p}$-thin subgroups can be described in terms of unmarked Cayley graph of $(G, S)$ :

Remark 5.4. $p=2, q=1, \mu$ is symmetric measure on $G$. Fix a sequence $\alpha_{i}$, tending to infinity. An element $g$ belongs to the subgroup $H_{\mu, 2,1, \alpha}$ if and only if

$$
\left.\left(\mu^{2 n}(e)-\mu^{* 2 n}(g)\right) / \alpha_{n}^{2}\right) \rightarrow 0
$$

as $n \rightarrow 0$. In particular, if $\mu$ is equidistributed on a finite symmetric generating set $S$, subgroups $H_{\mu, 2,1, \alpha_{i}}$ are defined by unmarked Cayley graph of $(G, S)$.

Proof. Observe that $\left|g \mu^{* n}-\mu^{* n}\right|_{2}^{2}=\left|\mu^{* n}\right|_{2}^{2}+\left|g \mu^{* n}\right|_{2}^{2}-2\left\langle\mu^{* n}, g \mu^{* n}\right\rangle=2\left|\mu^{* n}\right|_{2}^{2}-$ $2\left\langle\mu^{* n}, g \mu^{* n}\right\rangle=2\left(\sum_{x \in G}\left(\mu^{* n}(x)\right)^{2}-\sum_{x \in G} \mu^{* n}(x) \mu^{* n}(g x)\right)$, Since $\mu$ is symmetric, this is equal to $2\left(\sum_{x \in G} \mu^{* n}(x) \mu^{* n}\left(x^{-1}\right)-\sum_{x \in G} \mu^{* n}(g x) \mu^{* n}\left(x^{-1}\right)=2\left(\mu^{2 n}(e)-\mu^{* 2 n}(g)\right)\right.$. If $\mu$ is equidistributed on a finite symmetric generating set $S$, observe that $\mu^{* 2 n}(e)$ and $\mu^{* 2 n}(g)$ are defined by the unmarked Cayley graph of $(G, S)$ and the vertex in this Cayley graph corresponding to $g$.

Remark 5.5. In a particular case when $q=1, p=2$ and $G$ is non-amenable, the main $\ell_{2}$ thin subgroup in 5.1] coincides with the group, studied by Elder and Rogers in [8]. However, if $q=1, p=2$ and $G$ is amenable, the group defined in the above cited paper coincides with $G$, while the main $\ell_{2}$-thin subgroup $H_{\mu, p}$ is never equal to $G$ (for any infinite group $G)$. 
Now assume that $\mu$ has finite support, and consider the mappings $b_{\mu, U}^{p, q}$, defined in the introduction. Namely, for any non-principal ultrafilter $U$ on $\mathbb{N}$, put $\alpha^{p, q}(n)=\max _{s \in S} \|\left(\mu^{* n}\right)^{q}-$ $s\left(\mu^{* n}\right)^{q} \|_{p}$ and define the cocycle $b_{\mu, U}^{p, q}: G \rightarrow \ell_{p}(G)^{U}$ by

$$
b_{\mu, U}^{p, q}(g)=\left[\alpha^{p, q}(n)^{-1}\left(\left(\mu^{* n}\right)^{q}-g\left(\mu^{* n}\right)^{q}\right)\right]_{n} \in \ell_{p}(G)^{U} .
$$

The cocycle $b_{\mu, U}^{p, q}$ is independent, modulo scalar multiple, of the choice of the finite generating subset $S$. We note that $\ell_{p}(G)^{U}$ is an abstract $L_{p}$-space on which $G$ acts isometrically. Hence $b_{\mu, U}^{p, q}(G)$ is contained in a $G$-invariant separable $L_{p}$-subspace of $\ell_{p}(G)^{U}$.

Lemma 5.6. 1) [Direct products, $q=0, p \geq 1$ ] Let $G$ be a direct product of $A$ of subexponential growth and $B$ of exponential growth, and let $\mu=\mu_{A} \times \mu_{B}$ where $\mu_{A}(e)>$ 0 . Then there exists a subsequence $n_{i}$ such that subgroup $H_{\mu, 0, p}(G)=H_{\mu, p, 0}(G)$ contains A. Moreover, for any $n_{i}$ as above, any ultrafilter $U$ such that $U\left(n_{i}\right)=1$ for $q=0$ and $p \geq 1$ satisfy $b_{U, \mu}^{p, q}=b_{U, \mu_{B}}^{p, q}$.

2) [Direct product, $p=q=1$ ] Let $G$ be a direct product of a group A and B; let $\mu_{A}$, $\mu_{B}$ be non-degenerate measures on $A$ and $B$ such that the Poisson boundary of a random walk $\left(A, \mu_{A}\right)$ is trivial and Poisson boundary of $\left(B, \mu_{B}\right)$ is non-trivial. Put $\mu=\mu_{A} \times \mu_{B}$. Then for any choice of $n_{i}$ the main $\ell_{1}$-thin subgroup $H_{\mu, 1,1}(G)$ contains $A$. Moreover, for any ultrafilter $U$ it holds $b_{U, \mu}^{1,1}=b_{U, \mu_{B}}^{1,1}$.

3) [Direct products, $q=1, p=2$ ] Let $G$ be a direct product of an amenable $A$ and non-amenable group $B, \mu=\mu_{A} \times \mu_{B}$. Then for any $n_{i}$, the main $\ell_{2}$-thin subgroup $H_{\mu, 1,2}(G)=H_{\mu, 2,1}(G)$ contains $A$. Moreover, for any ultrafilter $U$ it holds $b_{U, \mu}^{2,1}=b_{U, \mu_{B}}^{2,1}$.

Proof. First we prove the claims of 1), 2), 3) about $\ell_{p}$-thin subgroups. Observe that since $B$ is of exponential growth, for any finite set $S$ there exists $v>1$ such that $v_{G, S}(n) \geq$ $v^{n}$ for all $n$. This implies that for each finite generating set $S_{B}$ of $B$ and each $C_{1}<1$ there exists $C_{2}>0$ such that for all $n$ at least $C_{1} n$ among balls of radius $i=1, \ldots, n$ have boundary greater than $C_{2} v_{B, S_{B}}(i)$. (Indeed, otherwise $v_{B, S_{B}}(n) \leq R_{B}^{n\left(1-C_{1}\right)}\left(1+C_{2}\right)^{C_{1} n}$, where $R_{B}$ denotes the cardinality of $B, S_{B}$, and taking $C_{1}$ close to 1 and $C_{2}$ close to 0 we would get a contradiction).

Since $A$ is of subexponential growth, for each $C$ and any $\epsilon_{1}, \epsilon_{2}>0$ at least $\left(1-\epsilon_{2}\right) n$ among the the balls of radius $i=1, \ldots, n$ have boundary at most $\epsilon_{2} v_{A}(i)$.

Consider a generating set $S=S_{A} \times S_{B}$, where $S_{A}, S_{B}$ are generating sets of $A, B$ respectively. We have $B_{S}(i)=B_{S_{A}}(i) \times B_{S_{B}}(i)$. Here $B_{G, S}(i)$ denotes the ball of radius $i$ in $G, S$. Observe also that for $S \in S_{A}$ it holds $s B_{G, S}(i) \backslash B_{G, S}(i)=s\left(B_{A, S_{A}}(i) \times\right.$ $\left.B_{B, S_{B}}(i)\right) \backslash\left(B_{A, S_{A}}(i) \times B_{B, S_{B}}(i)\right)=\left(s B_{A, S_{A}}(i) \backslash B_{A, S_{A}}(i)\right) \times B_{B, S_{B}}(i)$, and the cardinality of this set is at most $2\left(v_{A, S_{A}}(i)-v_{A, S_{A}}(i-1)\right) v_{B, S_{B}}(i)$, and with the same argument the cardinality of $s B_{G, S}(i) \backslash B_{G, S}(i)$, for $s \in S_{B}$ is at least $2 /\left|S_{B}\right|\left(v_{B, S_{B}}(i)-\right.$ $v_{B, S_{B}}(i-1) v_{A, S_{A}}(i)$ for some $s \in S_{B}$. This shows that there exists a sequence $n_{i}$, tending to infinity, such that

$$
\frac{v_{A, S_{A}}\left(n_{i}\right)-v_{A, S_{A}}\left(n_{i}-1\right)}{v_{A, S_{A}}\left(n_{i}\right)} \frac{v_{B, S_{B}}\left(n_{i}\right)}{v_{B, S_{B}}\left(n_{i}\right)-v_{B, S_{B}}\left(n_{i}-1\right)}
$$

tends to 0 as $i$ tends to infinity. By Remark 5.2 we know that for any group it holds $H_{\mu, 0, p}(G)=H_{\mu, p, 0}(G)$. Note that for any $n_{i}$ as above the this thin subgroup $H_{\mu, 0, p}(G)=$ $H_{\mu, p, 0}(G)$ with respect to a subsequence $n_{i}$ contains all $s \in S_{A}$. Therefore, in this case this subgroup contains $A$.

2) We recall that $\mu^{* n}=\mu_{A}^{* n} \mu_{B}^{* n}$. Take $a \in A$. Observe that $a \mu^{* n}-\mu^{* n}=\left(\mu_{A}^{* n}-\right.$ $\left.a \mu_{A}^{* n}\right) \mu^{* n}(B)$. It holds therefore $\left\|\left(\mu^{* n}-a \mu^{* n}\right)\right\|_{1}=\left\|\left(\mu_{A}^{* n}-a \mu_{A}^{* n}\right)\right\|_{1}$. Since the nondegenerate walk $\left(A, \mu_{A}\right)$ has trivial Poisson-Furstenberg boundary, for any $a \in A$ it holds 
$\left\|\left(\mu_{A}^{* n}-a \mu_{A}^{* n}\right)\right\|_{1} \rightarrow 0$ as $n$ tends to $\infty$, and therefore $\left\|\left(\mu^{* n}-a \mu^{* n}\right)\right\|_{1} \rightarrow 0$ as $n$ tends to $\infty$ (see Kaimanovich Vershik [20]). The above mentioned characterization also shows that since the Poisson boundary of $\left(B, \mu_{B}\right)$ is non-trivial, there exists $b \in B$ such that $\left\|\left(\mu_{B}^{* n}-b \mu_{B}^{* n}\right)\right\|_{1} \geq c>0$, and hence $\left\|\left(\mu^{* n}-b \mu^{* n}\right)\right\|_{1} \geq c>0$ for some positive constant $c$ and all $n$.

3) For $g=\left(g_{1}, g_{2}\right), g_{1} \in A, g_{2} \in B, \mu^{* n}\left(g_{1}, g_{2}\right)=\mu_{A}^{* n}\left(g_{1}\right) \mu_{B}^{* n}\left(g_{2}\right)$. For $h \in$ $A, \mu^{* n}\left(h\left(g_{1}, g_{2}\right)\right) / \mu^{* n}\left(g_{1}, g_{2}\right)=\mu_{A}^{* n}\left(h g_{1}\right) / \mu_{A}^{* n}\left(g_{1}\right) \rightarrow 1$ as $n \rightarrow \infty$, by [1] since $A$ is amenable [1]. Analogously, for $h \in B$ it holds $\mu^{* n}\left(h\left(g_{1}, g_{2}\right)\right) / \mu^{* n}\left(g_{1}, g_{2}\right)=$ $\mu_{B}^{* n}\left(h g_{2}\right) / \mu_{B}^{* n}\left(g_{2}\right) \rightarrow C_{h}$, where $C_{h} \neq 1$ for some $h$ among generators of $B$, since $B$ is non-amenable [1]. This implies that the scaling sequence $\alpha(n)$ is equivalent up to multiplicative constant to $\mu^{* n}(e)=\mu_{A}^{* n}(e) \mu_{B}^{* n}(e)$. Using Remark 5.4 we conclude that for all $s \in S_{A}\left\|s \mu^{* n}-\mu^{* n}\right\|_{2} / \alpha(n) \rightarrow 0$, and hence any $s \in A, s$ belongs to the $\ell_{2}$ thin subgroup for $q=1, p=2$. By Remark 5.3 we know that under assumption of 3) it holds $H_{\mu, 1,2}(G)=H_{\mu, 2,1}(G)$.

Now to prove the claims about the cocycles, take $g=(a, b) \in A \times B$, put $g^{\prime}=(e, b)$ and $g^{\prime \prime}=(a, e)$. It holds $g=g^{\prime} g^{\prime \prime}$. Under the assumption on $p$ and $q$ in 1), 2), 3) observe that

$$
\begin{gathered}
\left\|\left(\mu^{* n_{i}}\right)^{q}-g^{\prime}\left(\mu^{* n_{i}}\right)^{q}\right\|_{p}-\left\|\left(\mu^{* n_{i}}\right)^{q}-g^{\prime \prime}\left(\mu^{* n_{i}}\right)^{q}\right\|_{p} \leq\left\|\left(\mu^{* n_{i}}\right)^{q}-g\left(\mu^{* n_{i}}\right)^{q}\right\|_{p} \leq \\
\leq\left\|\left(\mu^{* n_{i}}\right)^{q}-g^{\prime}\left(\mu^{* n_{i}}\right)^{q}\right\|_{p}+\left\|\left(\mu^{* n_{i}}\right)^{q}-g^{\prime \prime}\left(\mu^{* n_{i}}\right)^{q}\right\|_{p}
\end{gathered}
$$

and that $\left\|\left(\mu^{* n_{i}}\right)^{q}-g^{\prime}\left(\mu^{* n_{i}}\right)^{q}\right\|_{p}=\left\|\left(\mu_{B}^{* n_{i}}\right)^{q}-g^{\prime}\left(\mu_{A}^{* n_{i}}\right)^{q}\right\|\left\|_{p}\right\| \mu_{A}^{n_{i}} \|_{p}$. This allows us to use 1) of Remark 5.7 and completes the proof of the Lemma.

Remark 5.7. $G=A \times B, \mu=\mu_{A} \times \mu_{B}, S=S_{A} \times S_{B}, S_{A}$ and $S_{B}$ are finite generating sets of $A$ and $B$.

1) Let $\alpha_{G}^{p, q}(n)$ be the maximal $\ell_{p}$ norm of $\left(\mu^{* n}\right)^{q}-s\left(\mu^{* n}\right)^{q}$, where the maximum is taken over $s \in S$; and let $\alpha_{A}^{p, q}(n)$ be the maximal $\ell_{p}$ norm of $\left(\mu_{A}^{* n}\right)^{q}-s\left(\mu_{A}^{* n}\right)^{q}$, the maximum is over $s \in S_{A}$ and $\alpha_{B}^{p, q}(n)$ is defined analogously. Let $\tilde{\theta}_{\mu}(n)$ be equal to $\alpha_{G}^{p, q}(n)$ divided by the $\ell_{p}$ norm of $\left(\mu^{* n}\right)^{q}$. If $\tilde{\theta}_{A}^{p, q}\left(n_{i}\right) / \tilde{\theta}_{B}^{p, q}\left(n_{i}\right)$ tends to zero for some sequence $n_{i}$ and $U$ is a non-principal ultrafilter such that $U\left(\left\{n_{i}\right\}\right)=1$, then $b_{\mu, U}^{p, q}=b_{\mu_{B}, U}^{p, q}$.

2) Take $q=1, p=2$. Put $\theta(n):=\left(\mu^{* 2 n}-\mu^{* 2 n+1}\right) / \mu^{* 2 n}$. Then $\theta(n)=\tilde{\theta}_{\mu}(n)^{2}$. In particular, if $\theta_{A}\left(n_{i}\right) / \theta_{B}\left(n_{i}\right)$ tends to zero and $U$ is a non-principal ultrafilter such that $U\left(\left\{n_{i}\right\}\right)=1$, then the corresponding harmonic cocycle is defined by that of $B$, that is $b_{\mu, U}=b_{\mu_{B}, U}$.

Remark 5.8. The fact that $A \times B, A$ is of subexponetial growth, $B$ is of exponential growth, satisfy the claim of 1), Lemma 5.6 not only for some sequence $n_{i}$ but for all sequences can be shown to be equivalent to a positive answer to both following questions $A$ ): is it true that no subset of balls is a Foelner sequence in A? B): Is it true that all balls form a Foelner sequence in $A$ ?

To our knowledge, it is not known whether to answer to A) is positive for all groups of exponential growth (this question is mentioned e.g. in [34]), and whether the answer to B) is positive for all groups of subexponential growth.

Example 5.9 (Dependance of $\ell_{p}$-thin subgroups on $p$ ). Let $G=F_{m} \times \mathbb{Z}^{d} 2 A$, where $m \geq 2, d \geq 3$ and $A$ is a finite group containing at least two elements. Let $\mu$ be a nondegenerate symmetric finitely supported measure. Then $\ell_{2}$-thin subgroup is not equal to $\ell_{1}$-thin subgroup. 
Proof. Observe that the $\ell_{2}$-thin subgroup $H_{\mu, 1,2}=H_{\mu, 2,1}$ contains $\mathbb{Z}^{d}\{A$ by 3 ) of Lemma 5.6 (in fact, it is equal to $\mathbb{Z}^{d} \curlyvee A$ ), while there exists $g \in \mathbb{Z}^{d} \nmid A$ which does not belong to $\ell_{1}$-thin subgroup since the Poisson boundary of $\mathbb{Z}^{d} \supsetneq A$ is non-trivial.

Remark 5.10. Let $G=C\{A$, where $C$ is an infinite group of at least cubic growth and $A$ is a finite group containing at least two elements. Let $\mu$ be a symmetric finitely supported "switch-walk-switch" measure on $G$. One can show that $H_{\mu, 1,1}$ is a finite subgroup of $G$. One can also show that for any integer $k \geq 0$ there exists $\mu$ as above such that $H_{\mu, 1,1}$ is isomorphic to $A^{m}$. In particular, this main $\ell_{1}$ thin subgroup $H_{\mu, 1,1}$ depends on the choice of a finitely supported symmetric measure $\mu$ and this subgroup is not normal.

Proof of Theorem C. Assume $d=2$ (the general case $d \geq 2$ is analogous).

We construct $G_{1}$ and $G_{2}$ as piecewise-automatic groups with returns of automata $\tau_{1}$, $\tau_{2}$, where $\tau_{1}, \tau_{2}: A \times X \rightarrow A$, the group generated by $\left(A, \tau_{1}\right)$ is of intermediate growth, $\tau_{2}: A \times X \rightarrow A$, the group $H_{2}$ generated by $\left(A, \tau_{2}\right)$ is non-amenable, and the action of $A$, considered as generators of $H$, is contracting for the action of $\tau_{1}$ for each brach of the rooted tree (see [9]).

More precisely, we chose automata $\tau_{1}$ and $\tau_{2}$ with the following properties: $\tau_{2}$ is a finite state automaton, containing $e, a, b, c, d$ as its states, such that $e$ acts trivially and $a, b, c, d$ generate the free product $\mathbb{Z} / 2 \mathbb{Z} *(\mathbb{Z} / 2 \mathbb{Z}+\mathbb{Z} / 2 \mathbb{Z})$ in the group generated by $\tau_{2}$.

If the states of $\tau_{2}$ are $e, a, b, c, d$ and the alphabet is 0,1 , we take as $\tau_{1}$ the standard finite state automaton for the first Gigorchuk group $(A=\{e, a, b, c, d\}, X=\{0,1\}$. In this case we can take as $G_{1}$ and $G_{2}$ either piecewise-automatic group or a piece-wise automatic group with returns defined by $\tau_{1}, \tau_{2}$ and $t_{i}, T_{i}, i \geq 1, T_{i-1}<t_{i}<T_{i}$. We do not know if $\tau_{2}$ as above exists, and therefore we consider as in [9] an automaton $\tau_{2}$ with the space of states possibly larger than $e, a, b, c, d$ (such automata exist by the result of Olijnyk [27], that shows that any free product of finite groups imbeds in a group generated by a finite state automaton), and we take as $\tau_{1}$ the standard finite state automaton for the first Grigorchuk group, (extended to some larger alphabet than 0 and 1 if the alpaheth of $\tau_{2}$ contains more than two letters) and consider the corresponding piecewise automatic group with returns $G_{\tau_{1}, \tau_{2}}\left(t_{i}, T_{i}\right)$.

To construct $G_{1}$ and $G_{2}$, we fix $\tau_{1}, \tau_{2}$ and construct sequences $t_{i}^{1}, T_{i}^{1}$ and $t_{i}^{2}, T_{i}^{2}\left(T_{i-1}^{1}<\right.$ $t_{i}^{1}<T_{i}^{1}, T_{i-1}^{2}<t_{i}^{2}<T_{i}^{2}$ ) by a simultaneous inductive procedure and we put $G_{1}=$ $G_{\tau_{1}, \tau_{2}}\left(t_{i}^{1}, T_{i}^{1}\right)$ and $G_{2}=G_{\tau_{1}, \tau_{2}}\left(t_{i}^{2}, T_{i}^{2}\right)$.

We need the following properties of piece-wise autmatic group with returns $G_{\tau_{1}, \tau_{2}}\left(t_{i}, T_{i}\right)$ (see proof of Proposition 1 in [9]). There exist $\Psi: \mathbb{N} \rightarrow \mathbb{N}$ and for each $i$ there exist "comparison groups" $\mathcal{A}\left(t_{1}, T_{1}, t_{2}, T_{2}, \ldots t_{i}\right)$ and $\mathcal{B}\left(t_{1}, T_{1}, t_{2}, T_{2}, \ldots t_{i}, T_{i}\right)$, such that the following holds for all non-decreasing sequences $t_{i}, T_{i}$ :

(1) all groups $\mathcal{A}\left(t_{1}, T_{1}, t_{2}, T_{2}, \ldots T_{i-1}\right)$ have a finite index subgroup which imbeds as a subgroup in a finite direct power of the the first Grigorchuk group $G_{1}$ (generated by $\left(A, \tau_{1}\right)$,

(2) all groups $\mathcal{B}\left(t_{1}, T_{1}, t_{2}, T_{2}, \ldots T_{i-1}, t_{i}\right)$ have a finite index subgroup which admits a surjective homomorphism to the group, generated by the automaton $\left(A, \tau_{2}\right)$,

(3) the balls of radius $\Psi\left(t_{i}\right)$ in $G\left(t_{1}, t_{2}, \ldots, T_{1}, T_{2}, \ldots\right)$ and $\mathcal{A}\left(t_{1}, T_{1}, t_{2}, T_{2}, \ldots t_{i-1}, T_{i-1}\right)$ coincide,

(4) the balls of radius $\Psi\left(T_{i}\right)$ in $G\left(t_{1}, t_{2}, \ldots, T_{1}, T_{2}, \ldots\right)$ and $\mathcal{B}\left(t_{1}, T_{1}, t_{2}, T_{2}, \ldots T_{i-1}, t_{i},\right)$ coincide.

Let $G, S_{G}, H, S_{H}$ be finitely generated groups such that the balls of radius $R+C$ in the marked Cayley graphs of $G, S_{G}, H, S_{H}$ coincide. Let $\mu_{H}$ and $\mu_{G}$ are measures which 
are equal after the identifications of these balls and such that $l_{G}(s) \leq C$ for any $s$ in the support of $\mu_{G}$. Observe that for any $n \leq R$ the scaling functions in the definition of $\ell_{p}$-thin subgroups are equal : $\alpha_{G, \mu_{G}, p}(n)=\alpha_{H, \mu_{H}, p}(n), \alpha_{G, \mu_{G}, p}^{\prime}(n)=\alpha_{H, \mu_{H}, p}^{\prime}(n)$ and for each $g$ in the ball of radius $C$ in the Cayley graph of $\left(G, S_{G}\right) \ell_{p}$ norms of $g\left(\mu_{G}^{* n}\right)^{q}-\left(\mu_{G}^{* n}\right)^{q}$ are equal to the $\ell_{p}$ norm of $h\left(\mu_{H}^{* n}\right)^{q}-\left(\mu_{H}^{* n}\right)^{q}$ for $h$ being the corresponding element in the ball of radius $C$ of $\left(H, S_{H}\right)$.

Suppose that we have chosen already $t_{1}^{1}, T_{1}^{1}, t_{2}^{1}, T_{2}^{1}, \ldots T_{i-1}^{1}$ and $t_{1}^{2}, T_{1}^{2}, t_{2}^{1}, T_{2}^{2}, \ldots t_{i}^{2}$. For any $\epsilon>0$ there exist $M_{i}$ such that for all $M_{i}^{\prime}>M_{i}$ there exists $M_{i}^{*}$ with the following property. For any $t_{i}^{1}>M_{i}^{*}$ and $T_{i}^{2}>M_{i}^{*}$ and any $n: M_{i}<n<M_{i}^{\prime}$ the ratio of $\ell_{p}$ norms $s_{1}\left(\mu^{* n}\right)^{q}-\left(\mu^{* n}\right)^{q}$ and $s_{2}\left(\mu^{* n}\right)^{q}-\left(\mu^{* n}\right)^{q}$ in $G=G_{1} \times G_{2}$ is smaller than $\epsilon$ for all $s \in S_{1}$ and some $s \in S_{2}$.

To prove this, we combine the observation about Cayley graphs above with the claims 1), 2), 3) of Lemma 5.6, for $\mathcal{A}=\mathcal{A}\left(t_{1}^{1}, T_{1}^{1}, t_{2}^{1}, T_{2}^{1}, \ldots T_{i-1}^{1}\right), \mathcal{B}=\mathcal{B}\left(t_{1}^{2}, T_{1}^{2}, t_{2}^{1}, T_{2}^{2}, \ldots t_{i}^{2}\right)$. Tthe group $A$ is of intermediate growth and hence this group is amenable and finitely supported random walks have trivial boundary, $B$ has a finite index subgroup sujecting to a non-amenable group, and hence non-amenable.

Now suppose that we have chosen already $t_{1}^{1}, T_{1}^{1}, t_{2}^{1}, T_{2}^{1}, \ldots t_{i}^{1}$ and $t_{1}^{2}, T_{1}^{2}, t_{2}^{1}, T_{2}^{2}, \ldots t_{i}^{2}, T_{i}^{2}$. For any $\epsilon>0$ there exist $N_{i}$ such that for all $N_{i}^{\prime}>N_{i}$ there exists $N_{i}^{*}$ with the following property. For any $T_{i}^{1}>N_{i}^{*}$ and $t_{i+1}^{2}>M_{i}^{*}$ and any $n: N_{i}<n<N_{i}^{\prime}$ the ratio of $\ell_{p}$ norms of $s_{2}\left(\mu^{* n}\right)^{q}-\left(\mu^{* n}\right)^{q}$ and $s_{1}\left(\mu^{* n}\right)^{q}-\left(\mu^{* n}\right)^{q}$ in $G=G_{1} \times G_{2}$ is smaller than $\epsilon$ for all $s_{2} \in S_{2}$ and some $s_{1} \in S_{1}$.

This implies that for some choice of $t_{i}^{1}, T_{i}^{1}$ and $t_{i}^{2}, T_{i}^{2}$ there exist sequences $n_{i}, m_{i}$ tending to infinity, such that the following holds. The ratio of $\ell_{p}$ norms of $s_{1}\left(\mu^{* n_{i}}\right)^{q}-$ $\left(\mu^{* n_{i}}\right)^{q}$ and the scaling sequence $\alpha\left(n_{i}\right)$ tend to 0 for all $s_{1} \in S_{1}$. This implies that all $s_{1} \in S_{1}$, as well as all $g \in G_{1}$ belong to the main $\ell_{p}$ thin subgroup $H_{\mu, p, q}$, corresponding to $n_{i}$. The ratio of $\ell_{p}$ norms of $s_{2}\left(\mu^{* m_{i}}\right)^{q}-\left(\mu^{* m_{i}}\right)^{q}$ and the scaling sequence $\alpha\left(m_{i}\right)$ tend to 0 for all $s_{2} \in S_{2}$. This implies that all $s_{2} \in S_{e}$, as well as all $g \in G_{2}$ belong to the main $\ell_{p}$ thin subgroup $H_{\mu, p, q}$, corresponding to $m_{i}$. Consider an ultrafilter $U_{m}$ such that $U\left(m_{i}\right)=1$ and an ultrafilter $U_{n}$ such that $U\left(n_{i}\right)=1$. Using 1), 2), 3) of Lemma5.6 we also observe that $b_{\mu, U_{n}}^{p, q}$ is equal to $b_{\mu_{2}, U_{n}}^{p, q}$ and that $b_{\mu, U_{m}}^{p, q}$ is equal to $b_{\mu_{1}, U_{m}}^{p, q}$.

Corollary 5.11. Let $G_{i}, \mu_{i}$ be as in the formulation of Theorem $\square$ Take $q=0,1$ or 2 and $p=1$ or 2 . For each $j: 1 \leq j \leq D$ there exists $n_{i, j}$ such that for all the main $\ell_{p}$-thin subgroup $H_{\mu, p, q}$ of $G$ with respect to $n_{i}=n_{i, j}$ contains $\prod_{k: k \neq j} G_{k}$. In particular, there exist at least $D$ not equal $\ell_{p}$-thin subgroups.

\section{REFERENCES}

[1] André Avez, Limite de quotients pour des marches aléatoires sur des groupes, C. R. Acad. Sci. Paris Sér. A-B 276 (1973), A317-A320 (French). MR0315750

[2] Bachir Bekka, Pierre de la Harpe, and Alain Valette, Kazhdan's property (T), New Mathematical Monographs, vol. 11, Cambridge University Press, Cambridge, 2008. MR2415834

[3] Patrick Billingsley, Probability and measure, 3rd ed., Wiley Series in Probability and Mathematical Statistics, John Wiley \& Sons, Inc., New York, 1995. A Wiley-Interscience Publication. MR1324786

[4] Marc Burger and Shahar Mozes, Lattices in product of trees, Inst. Hautes Études Sci. Publ. Math. 92 (2000), 151-194 (2001). MR1839489

[5] Yves de Cornulier, Fixed point and almost fixed point, after Gromov and V.Lafforque (http://www.normalesup.org/ cornulier/grolaf.pdf).

[6] Patrick Delorme, 1-cohomologie des représentations unitaires des groupes de Lie semi-simples et résolubles. Produits tensoriels continus de représentations, Bull. Soc. Math. France 105 (1977), no. 3, 281-336 (French). MR0578893 
[7] A. Dvoretzky and P. Erdös, Some problems on random walk in space, Proceedings of the Second Berkeley Symposium on Mathematical Statistics and Probability, 1950., University of California Press, Berkeley and Los Angeles, 1951, pp. 353-367. MR0047272

[8] Murray Elder and Cameron Rogers, Random walks and amenability of groups (https://arxiv.org/abs/1605.04065).

[9] Anna Erschler, Piecewise automatic groups, Duke Math. J. 134 (2006), no. 3, 591-613, DOI 10.1215/S00127094-06-13435-X. MR2254627

[10] A. Erschler, Almost invariance of distributions for random walks on groups, preprint, arXiv:1603.01458

[11] David Fisher and Gregory Margulis, Almost isometric actions, property (T), and local rigidity, Invent. Math. 162 (2005), no. 1, 19-80, DOI 10.1007/s00222-004-0437-5. MR2198325

[12] R. I. Grigorchuk, Degrees of growth of finitely generated groups and the theory of invariant means, Izv. Akad. Nauk SSSR Ser. Mat. 48 (1984), no. 5, 939-985 (Russian). MR764305

[13] Mikhael Gromov, Groups of polynomial growth and expanding maps, Inst. Hautes Études Sci. Publ. Math. 53 (1981), 53-73. MR623534 (83b:53041)

[14] M. Gromov, Random walk in random groups, Geom. Funct. Anal. 13 (2003), no. 1, 73-146, DOI 10.1007/s000390300002. MR1978492

[15] _ Asymptotic invariants of infinite groups, Geometric group theory, Vol. 2 (Sussex, 1991), London Math. Soc. Lecture Note Ser., vol. 182, Cambridge Univ. Press, Cambridge, 1993, pp. 1-295. MR1253544

[16] Alain Guichardet, Sur la cohomologie des groupes topologiques. II, Bull. Sci. Math. (2) 96 (1972), 305-332 (French). MR0340464

[17] Y. Guivarc'h, Sur la loi des grands nombres et le rayon spectral d'une marche aléatoire, Conference on Random Walks (Kleebach, 1979), Astérisque, vol. 74, Soc. Math. France, Paris, 1980, pp. 47-98, 3 (French, with English summary). MR588157

[18] Naresh C. Jain and William E. Pruitt, The range of random walk, Proceedings of the Sixth Berkeley Symposium on Mathematical Statistics and Probability (Univ. California, Berkeley, Calif., 1970/1971), Univ. California Press, Berkeley, Calif., 1972, pp. 31-50. MR0410936

[19] Kate Juschenko and Nicolas Monod, Cantor systems, piecewise translations and simple amenable groups, Ann. of Math. (2) 178 (2013), no. 2, 775-787, DOI 10.4007/annals.2013.178.2.7. MR3071509

[20] V. A. Kaĭmanovich and A. M. Vershik, Random walks on discrete groups: boundary and entropy, Ann. Probab. 11 (1983), no. 3, 457-490. MR704539

[21] Harry Kesten, Symmetric random walks on groups, Trans. Amer. Math. Soc. 92 (1959), 336-354. MR0109367

[22] Nicholas J. Korevaar and Richard M. Schoen, Global existence theorems for harmonic maps to nonlocally compact spaces, Comm. Anal. Geom. 5 (1997), no. 2, 333-387, DOI 10.4310/CAG.1997.v5.n2.a4. MR1483983

[23] James R. Lee and Yuval Peres, Harmonic maps on amenable groups and a diffusive lower bound for random walks, Ann. Probab. 41 (2013), no. 5, 3392-3419, DOI 10.1214/12-AOP779. MR3127886

[24] Hiroki Matui, Some remarks on topological full groups of Cantor minimal systems, Internat. J. Math. 17 (2006), no. 2, 231-251, DOI 10.1142/S0129167X06003448. MR2205435

[25] Ngaiming Mok, Harmonic forms with values in locally constant Hilbert bundles, Proceedings of the Conference in Honor of Jean-Pierre Kahane (Orsay, 1993), 1995, pp. 433-453. MR1364901

[26] V. Nekrashevych, Palyndromic subshifts and simple periodic groups of intermediate growth, preprint, arXiv: 1601.01033

[27] A. S. Oliinnyk, Free products of finite groups and groups of finitely automatic permutations, Tr. Mat. Inst. Steklova 231 (2000), no. Din. Sist., Avtom. i Beskon. Gruppy, 323-331 (Russian, with Russian summary); English transl., Proc. Steklov Inst. Math. 4 (231) (2000), 308-315. MR1841761

[28] D. V. Osin, Exponential radicals of solvable Lie groups, J. Algebra 248 (2002), no. 2, 790-805, DOI 10.1006/jabr.2001.9036. MR1882124

[29] Narutaka Ozawa, A functional analysis proof of Gromov's polynomial growth theorem, preprint, arXiv: 1510.04223

[30] Hans Reiter and Jan D. Stegeman, Classical harmonic analysis and locally compact groups, 2nd ed., London Mathematical Society Monographs. New Series, vol. 22, The Clarendon Press, Oxford University Press, New York, 2000. MR1802924

[31] Yehuda Shalom, Harmonic analysis, cohomology, and the large-scale geometry of amenable groups, Acta Math. 192 (2004), no. 2, 119-185, DOI 10.1007/BF02392739. MR2096453 (2005m:20095)

[32] _ Rigidity of commensurators and irreducible lattices, Invent. Math. 141 (2000), no. 1, 1-54, DOI 10.1007/s002220000064. MR1767270 
[33] Yves Stalder, Fixed point properties in the space of marked groups, Limits of graphs in group theory and computer science, EPFL Press, Lausanne, 2009, pp. 171-182. MR2562144

[34] Romain Tessera, Volume of spheres in doubling metric measured spaces and in groups of polynomial growth, Bull. Soc. Math. France 135 (2007), no. 1, 47-64 (English, with English and French summaries). MR2430198

[35] Russ Thompson, The rate of escape for random walks on polycyclic and metabelian groups, Ann. Inst. Henri Poincaré Probab. Stat. 49 (2013), no. 1, 270-287, DOI 10.1214/11-AIHP455 (English, with English and French summaries). MR3060157

A.E.: C.N.R.S., DÉPARTEMENT DE MATHÉMATIQUES ET APPLICATIONS, ÉCOlE NoRMALE SUPÉRIEURE, PSL RESEARCh INSTITUTE, 45, RUE D'Ulm, 75005, PARIS, FRANCE

E-mail address: anna.erschler@ens.fr

N.O.: Research Institute of Mathematical Sciences, Kyoto University, KitashiRakawaOIWAKE, KYOTO 606-8502, JAPAN

E-mail address: narutaka@kurims.kyoto-u.ac.jp 